\title{
Diagnosing cross-scale kinetic energy exchanges from two submesoscale permitting ocean models.
}

\author{
Adekunle Ajayi ${ }^{1}$, Julien Le Sommer ${ }^{1}$, Eric P. Chassignet ${ }^{2}$, Jean-Marc \\ Molines $^{1}$, Xiaobiao $\mathbf{X u}^{2}$, Aurelie Albert ${ }^{1}$, and William Dewar ${ }^{1,2}$ \\ ${ }^{1}$ Universite Grenoble Alpes /CNRS/IGE, Grenoble, France \\ ${ }^{2}$ Florida State University, Tallahassee, USA.
}

Key Points:

-We used two submesoscale permitting ocean models of the North Atlantic Ocean to investigate kinetic energy exchanges at fine-scales.

- KE fluxes at fine-scales are strongly impacted by submesoscale turbulence with a stronger forward cascade in winter within the mixed-layer.

- Not accounting for ageostrophic motions yields a significant under-estimation of the forward cascade.

Corresponding author: Adekunle Ajayi, adekunle.ajayi@univ-grenoble-alpes.fr

This article has been accepted for publication and ${ }^{-1}$ undergone full peer review but has not been through the copyediting, typesetting, pagination and proofreading process, which may lead to differences between this version and the Version of Record. Please cite this article as doi: 10.1029/2019MS001923.

This article is protected by copyright. All rights reserved. 


\section{Abstract}

Fine-scale motions $(<100 \mathrm{~km})$ contribute significantly to the exchanges and dissipation of kinetic energy in the upper ocean. However, knowledge of ocean kinetic energy at finescales (in terms of density and transfers) is currently limited due to the lack of sufficient observational datasets at these scales. The sea-surface height measurements of the upcoming SWOT altimeter mission should provide information on kinetic energy exchanges in the upper ocean down to 10-15 km. Numerical ocean models, able to describe ocean dynamics down to $\sim 10 \mathrm{~km}$, have been developed in anticipation of the SWOT mission. In this study, we use two state-of-the-art, realistic, North Atlantic simulations, with horizontal resolutions $\sim 1.5 \mathrm{~km}$, to investigate the distribution and exchanges of kinetic energy at fine-scales in the open ocean. Our results show that the distribution of kinetic energy at fine-scales approximately follows the predictions of quasi-geostrophic dynamics in summertime but is somewhat consistent with submesoscale fronts-dominated regimes in wintertime. The kinetic energy spectral fluxes are found to exhibit both inverse and forward cascade over the top $1000 \mathrm{~m}$, with a maximum inverse cascade close to the average energy-containing scale. The forward cascade is confined to the ocean surface and shows a strong seasonality, both in magnitude and range of scales affected. Our analysis further indicates that highfrequency motions ( $<1$ day) play a key role in the forward cascade and that the estimates of the spectral fluxes based on geostrophic velocities fail to capture some quantitative aspects of kinetic energy exchanges across scales.

\section{Plain Language Summary}

The dynamics of oceanic motions with scales $<100 \mathrm{~km}$ (fine-scales) are currently not well known. This is due to the lack of sufficient observational datasets at these scales in the ocean. There are suggestions from recent studies that this class of motions impacts the distribution and exchanges of kinetic energy in the ocean. To better understand fine-scale motions, the Surface Water and Ocean Topography (SWOT) satellite has been assembled. SWOT is expected for lunch in 2022 and will provide an unprecedented view of the ocean down to a wavelength of 10-15 km. In anticipation of the SWOT mission, numerical ocean models capable of resolving fine-scale oceanic motions have been designed and implemented. In this study, we use two of these simulations to investigate how kinetic energy is exchanged between oceanic motions at fine-scales. Our results show that submesoscale turbulence (a class of oceanic turbulence at fine-scale) and high-frequency motions affect the kinetic energy exchanges by providing a route to kinetic energy towards dissipation. Also, we found that kinetic energy exchanges based on the future SWOT dataset might underestimate the true magnitude of the transfer of kinetic energy towards finer scales. 


\section{Introduction}

The ocean is a turbulent fluid with a broad range of energetic scales, ranging from large $~$ $\mathrm{O}(1000 \mathrm{~km})$ to centimeter scales. The ocean kinetic energy is mostly concentrated in the quasi-geostrophic mesoscale eddy field with scales $\sim \mathrm{O}(100 \mathrm{~km})$ (Stammer \& Böning, 1992). Due to non-linear interactions among different length scales, energy can be transferred both from large to small (forward, or direct cascade) and from small to large scale (inverse cascade). Understanding the distribution of kinetic energy (KE) and variance across scales in oceanic flows is, therefore, key to our knowledge of ocean circulation (Ferrari \& Wunsch, 2009).

To estimate the variance and energy associated with eddy motions at different scales, velocity wavenumber power spectral density has proven to be very efficient (Le Traon et al., 1990, 2008; Fu et al., 2010; Dufau et al., 2016; Uchida et al., 2017). However, spectral density does not indicate the direction of kinetic energy exchanges between the different scales. A better knowledge of cross-scale energy exchanges is gained by looking at the KE cascade due to nonlinearity. This important feature in turbulence study dates back to the work of Charney (1971) and Salmon (1980) on geostrophic turbulence. For stratified rotating quasi-two-dimensional fluid motion, classical geostrophic turbulence theory predicts a direct cascade of energy if the flow is depth-dependent (baroclinic) but an inverse cascade of energy if the flow is depth-independent (barotropic). In particular, for the ocean with a surface intensified stratification, energy from higher baroclinic modes concentrates in the first mode and then converges toward the scale of the Rossby radius of deformation $\left(R_{d}\right)$ (Smith \& Vallis, 2002). At $R_{d}$, baroclinic energy is converted to barotropic mode via barotropization. At this point, most of the energy near the deformation scale cascade towards larger scales while a small fraction undergoes direct cascades to dissipation (see Figure 1).

This prediction of geostrophic turbulence theory has been observed both in numerical simulations and the real ocean but with a little discrepancy. Based on altimeter data, Scott and Wang (2005) showed that an inverse cascade of energy dominates the (Pacific) ocean at scales larger than $R_{d}$. So, if one agrees that the altimeter data is reflecting the first baroclinic mode (Smith \& Vallis, 2002), then this is in contrast with geostrophic turbulence theory which predicts a forward cascade for a baroclinic flow. Scott and Wang (2005) argued that there might be an inverse cascade associated with the first baroclinic mode and that this would only partially reduce the forward flux of total baroclinic energy. This total energy forward flux is the source of the kinetic energy that arrives near the deformation scale from the large-scale mean flow via baroclinic instability. From this discrepancy, two questions arise. (i) is the inverse cascade seen at the surface due to the barotropic mode? or (ii) is it possible that the baroclinic modes experience an inverse cascade? Scott and 
Arbic (2007) using a 2-layer model simulation showed that the inverse cascade at the ocean surface is mostly baroclinic with a small contribution from the barotropic mode. The results from Scott and Arbic (2007) are consistent with the proposed modification to geostrophic turbulence by Scott and Wang (2005). More recent literature (Schlösser \& Eden, 2007; Sasaki et al., 2017; Tulloch et al., 2011; Aluie et al., 2017; Brüggemann \& Eden, 2015; Kjellsson \& Zanna, 2017; Khatri et al., 2018) have also shown that an inverse cascade of energy mostly dominates the surface ocean at scales larger than $R_{d}$.

In contrast, little is known regarding energy cascade at scales $<R_{d}$, where oceanic motion is dominated by submesoscale motions $(<20-50 \mathrm{~km})$. Results from numerical simulation and observation have shown an injection of energy in winter at submesoscale (Sasaki et al., 2017). This energy injection is partly responsible for both meso and submesoscale seasonality (Uchida et al., 2017; Capet, Campos, \& Paiva, 2008; Sasaki et al., 2014) and has been argued to be associated with mixed layer instability (Callies, Ferrari, et al., 2015; Qiu et al., 2014; Sasaki et al., 2014; Brannigan et al., 2015; Rocha et al., 2016). This seasonality is responsible for the shallowing of $\mathrm{KE}$ spectral slope from -3 in summer to -2 in winter and is usually interpreted as a shift from turbulence dominated by interior gradients (Philips regime) to a regime dominated by surface driven turbulence (Charney regime) (Sasaki et al., 2014). Apart from the work of Sasaki et al. (2014, 2017); Schubert et al. (2020), we are unaware of any investigation on the implication of submesoscale seasonality on cross-scale energy exchanges at the basin scale and one of the objective of this study is to investigate how submesoscales modify cross-scale kinetic energy exchanges at fine-scales.

Submesoscale resolving ocean models have been developed in anticipation of the Surface Water and Ocean Topography (SWOT) satellite mission (Fu et al., 2010). On a global scale, satellite altimeters remain the primary source of information on the distribution of energy across scales. However, at the moment, the resolution capability of our existing oceanobserving satellite altimeters stands at roughly $70 \mathrm{~km}$ (Dufau et al., 2016). This limitation undermines our ability to investigate energy exchanges at scales $<100 \mathrm{~km}$. To solve this challenge, SWOT is implemented to provide ten times higher resolution than conventional altimeters, and numerical ocean models have been designed to prepare for SWOT. These state-of-the-art numerical experiments with high-resolution capability, thereby provide an opportunity to study cross-scale energy exchanges down to kilometric scales.

In this study, we aim to investigate the distribution and transfer of energy across different scales by using outputs of two submesoscale permitting ocean models of the North Atlantic. In particular, we focus on the seasonality and depth penetration of cross-scale $\mathrm{KE}$ variance and transfer with an emphasis on scales $<100 \mathrm{~km}$. This paper is organized as follows; section 2 presents a description of the two numerical simulations. In section 3 , 
we examine the kinetic energy wavenumber spectral density and slope. The KE cascade, its seasonality, and the role of high frequency and ageostrophic motions on the cascade are discussed in section 4. Finally, in section 5, we summarize the findings and discuss the relevance of this work in anticipation for SWOT mission.

\section{Numerical simulations of the North Atlantic Ocean}

In this study, we use numerical outputs from two submesoscale eddy-permitting simulations of the North Atlantic: a NEMO-based simulation with a horizontal resolution of $1 / 60^{\circ}$ (NATL60) and an HYCOM-based (HYbrid Coordinate Ocean Model) simulation with a horizontal resolution of $1 / 50^{\circ}$ (HYCOM50).

The NEMO-based NATL60 has a horizontal grid spacing ranging from $1.6 \mathrm{~km}$ at $26^{\circ} \mathrm{N}$ to $0.9 \mathrm{~km}$ at $65^{\circ} \mathrm{N}$. The initial and open boundary conditions are based on the GLORYS2v3 ocean reanalysis with a relaxation zone at the northern boundary for sea-ice concentration and thickness. The model has 300 vertical levels with a resolution of $1 \mathrm{~m}$ at the top-most layers. The grid and bathymetry follow Ducousso et al. (2017), while the atmospheric forcing is based on DFS5.2 (Dussin et al., 2018). DFS5.2 forcing is based on ERA-interim reanalysis. The spatial resolution of the atmospheric fields is 0.75 degrees. All variables used to compute turbulent fluxes (air temperature and humidity at $2 \mathrm{~m}$, wind velocity components at $10 \mathrm{~m}$ ) are 3-hourly. In order to implicitly adapt lateral viscosity and diffusivity to flow properties, a third-order upwind advection scheme is used for both momentum and tracers in the model simulation. The model was spun-up for six months, and a one-year simulation output from October 2012 to September 2013 is used in this study. The simulation output used in this study is the same as the one used in Amores et al. (2018), Buckingham et al. (2019) and Ajayi et al. (2020). An earlier version of this simulation set-up was used in Ducousso et al. (2017) and Fresnay et al. (2018).

NATL60 ocean simulation has been evaluated, using in situ observations in terms of the kinetic energy levels at different wavelengths (see Figure 1 of supporting information document (SI)). In terms of the dynamics of the resolved fine-scales in the upper ocean, results from Buckingham et al. (2019) show that the statistics of horizontal velocity tensor predicted by NATL60 agree reasonably well with observation (OSMOSIS datasets). However, their results also show that there is a likelihood of extreme divergent motions in OSMOSIS that is not captured by NATL60. That NATL60 underestimates divergent motions compared to observation isn't that surprising because NATL60 model simulation is without tidal forcing, one of the major sources of wave energy. The model, however, reproduces fairly well other forms of internal gravity waves (see Figure 2 of SI). 
The HYCOM-based HYCOM50 extends from $28^{\circ} \mathrm{S}$ to $80^{\circ} \mathrm{N}$ and has a horizontal grid spacing ranging from $2.25 \mathrm{~km}$ at the equator, $\sim 1.5 \mathrm{~km}$ in the Gulf Stream region, and 1 $\mathrm{km}$ in the subpolar gyre. As for NATL60, the effective resolution is about $10-15 \mathrm{~km}$. The vertical coordinate is hybrid and consists of 32 layers. The simulation is initialized using potential temperature and salinity from the GDEM climatology and spun up from rest for 20 years using climatological atmospheric forcing from ERA-40 (Uppala et al., 2005), with 3-hourly wind anomalies from the Fleet Numerical Meteorology and Oceanography Center 3-hourly Navy Operational Global Atmospheric Prediction System (NOGAPS) for the year 2003. The horizontal viscosity operator is a combination of Laplacian and Biharmonic. The bathymetry is based on the Naval Research Laboratory (NRL) digital bathymetry database. The model configuration and a detailed evaluation of the model results in the Gulf Stream region with observations are documented in Chassignet and Xu (2017).

In this study, we use the output from October 2012 to September 2013 for NATL60 and year 20 (last year of the simulation) for HYCOM50. Winter and summer correspond to January-February-March (JFM) and July-August-September (JAS), respectively. The summer analysis presented for NATL60 corresponds to that of the year 2013. Since NATL60 covers a smaller domain than HYCOM50, we consider the HYCOM50 outputs for the same region covered by NATL60 to have comparable results. To capture regional variability in the distribution of energy across scales, we perform spectral analysis in sub-domains of $1410^{\circ} \times 10^{\circ}$ boxes across the North Atlantic. We focus specifically on quantifying kinetic energy wavenumber spectral density (Eq. 1) and flux (Eq. 2) using horizontal velocity fields. In equation (1) and equation (2), ^ refers to Fourier transform, * represents the complex conjugate, $R e$ refers to the real part of a complex number and $k=\sqrt{k_{x}^{2}+k_{y}^{2}}$. Before performing spectral analysis, the $2 \mathrm{D}$ velocity field from each subdomain (box) is detrended in both directions, and a $50 \%$ cosine taper window (Tukey windowing) is applied for tapering. An FFT is applied to the tapered data, and a 1D isotropic spectrum is obtained by averaging in the azimuthal direction. Our spectral method is consistent with procedures previously used in Stammer and Böning (1992); Sasaki and Klein (2012); and Chassignet and $\mathrm{Xu}(2017)$.

$$
\begin{gathered}
E(k)=\int_{k}^{k+\delta k}\left[\widehat{\mathbf{u}}^{*} \cdot \widehat{\mathbf{u}}\right](k) d k \\
\Pi(k)=\int_{k}^{k_{s}}-\operatorname{Re}\left[\widehat{\mathbf{u}}^{*} \cdot\left(\mathbf{u} \cdot \widehat{\nabla}_{\mathbf{H}} \mathbf{u}\right)\right](k) d k
\end{gathered}
$$

Both NATL60 and HYCOM50 resolve the first Rossby radius of deformation everywhere within the model domain, and these simulations reproduce realistic eddy statistics with levels 
of kinetic energy in the range of altimetric observations (Chassignet and Xu (2017), see also Figure 2). A summary of the model parameters is tabulated in Table 1. Both simulations are submesoscale permitting ocean models and a discussion on the ability of these models to resolve the dynamics of submesoscales in terms of eddy length scale, submesoscale energy and their associated seasonality can be found in Ajayi et al. (2020).

We present in Figure 3, the root-mean-square of sea surface height computed from one year daily outputs of NATL60, HYCOM50, compared with AVISO. The AVISO SSH field is derived from observations obtained by altimeter missions and then interpolated onto a $0.25^{\circ}$ mercator grid. In this comparison, we have used AVISO mean dynamical topography dataset from October 2012 to September 2013. The SSH fields in Figure 3 from NATL60 and HYCOM50 have been resampled onto a $0.25^{\circ}$ grid to have comparable results. The SSH rms of the models and AVISO data appears to be consistent in terms of the geographical pattern of energetic oceanic motions except for the differences along the Gulf Stream. The Gulf Stream seems to be more energetic in HYCOM50 compared to NATL60 and AVISO. HYCOM50 has a spin-up of 20 years while NATL60 has a spin-up of 6 months. We hypothesize that HYCOM50 long spin-up allows for the full development of the Gulf Stream energetics and this difference in terms of spin-up could be contributing to differences in the overall energetics of the two simulations. The implication of the short spin-up for NATL60 is more obvious in the time evolution of the domain-averaged kinetic energy (see Figure 3 of SI). The kinetic energy is still increasing with time and is yet to attain equilibrium. A similar curve for HYCOM50 is available in Figure 2 of Chassignet and Xu (2017). The comparison of the KE spectral in summer of the year 2012 vs the year $2013^{1}$ (see Figure 4 of SI) further highlights the increase in energy (for NATL60) that is characterized by higher variance and larger eddies at low wavenumbers in year 2013.

The two simulations are similar but are not without differences. HYCOM50 appears to be more energetics compared to NATL60. The disparity between the two models' energy level is not the main focus of this paper, but we shall propose a few reasons why the two models could differ in terms of energetic. Firstly, we hypothesize that the eddy structures in NATL60 are not fully developed due to the short spin-up (6 months for NATL60 versus 20 years for HYCOM50). The first two years of the HYCOM50 simulation show an increase of total kinetic energy level; see Figure 2 in Chassignet and Xu (2017). Furthermore, the typical scale of eddies are smaller in NATL60 compared to HYCOM50 (Ajayi et al., 2020), and this could be a direct consequence of the shorter duration of the model spin-up. Secondly, the question did arise as to whether the coarser vertical resolution in HYCOM50

${ }^{1}$ The summer analysis presented in the article corresponds to that of year 2013 
(32 hybrid vertical layers versus 300 z-levels in NATL60) could lead to a stronger inverse cascade and hence a higher energy level because of an under-resolved stratification and the depth dependence of flows. A comparison of the vorticity spectral coherence as a function of depth shows that the two simulations are essentially identical in terms of the depth penetration of energetic eddy structures (Ajayi et al., 2020). Furthermore, in section 4, a comparison of the KE spectral flux at depths for the two simulations will show that the HYCOM50 upscale energy flux is not surface intensified and that having only 32 isopycnal vertical levels is not detrimental to the representation of the dynamics in the ocean interior. Thirdly, the choice of sub-grid parameterization is different between the two simulations and could have a substantial effect on how energy is dissipated in each model.

Up until recently, most basin-scale numerical models like those used in this study usually store simulation outputs in the form of daily averages due to limitations in storage and computational resources. This limitation comes with a caveat. Daily averaging the model outputs suppresses high-frequency motions ( $>f$, where $f$ is the Coriolis frequency). These motions are mostly dominated by ageostrophic motions that include unbalanced submesoscales and fast propagating internal gravity waves. There are new shreds of evidence, based on idealized simulations that suggest that high-frequency motions (particularly wave motions) can provide a route to kinetic energy dissipation (Barkan et al., 2017; Rocha et al., 2018). It would be interesting to investigate these new results in realistic simulations like NATL60 and HYCOM50. Unfortunately, NATL60 and HYCOM50 have most of their outputs stored in daily averages except for the last month of simulation for HYCOM50 and surface quantities for NATL60. To that end, the results presented in this study are based on daily averages of velocity fields from the two simulations. We have only used analysis based on hourly output where necessary to illustrate the impacts of high-frequency motions on the kinetic energy distribution and exchanges.

\section{Distribution of Kinetic Energy}

In this section, we discuss the variance at different scales of motions by analyzing the kinetic energy wavenumber power spectral density. In general, horizontal wavenumber spectral density exhibits power-law behavior, where the exponent is interpreted in terms of the dynamical processes governing the eddy energy transfer. Existing theoretical frameworks (for horizontal velocity at scale $>R d$ ) predict a spectral slope of -3 and $-5 / 3$ for $Q G$ and SQG (surface quasi-geostrophic) turbulence respectively. A slope of -2 is also well known for a front dominated flow (Shcherbina et al., 2013; Callies \& Ferrari, 2013). Over the years, many research works have tried to establish the accuracy of these predictions by using outputs of realistic ocean models (Sasaki \& Klein, 2012; Chassignet \& Xu, 2017; Uchida et 
al., 2017) and also recently within the context of the real ocean by using altimeter dataset (Le Traon et al., 1990; Dufau et al., 2016). Their results have argued for the non-existence of a universal wavenumber spectrum (Le Traon et al., 2008) following observed regional variability. We shall discuss in section 3.1, the distribution of kinetic energy as predicted by NATL60 \& HYCOM50 and in section 3.2 we discuss the variability associated with this distribution and their associated estimated slope.

\subsection{Spectral Density}

In Figure 4, we present the kinetic energy spectral density as a function of depth for the two simulations. For simplicity, we show this comparison only for Box 8, a box located at the center of the North Atlantic basin. In most of the regions, the peak of the spectral density is around the mesoscale motions (100-500 km). As expected, the energy associated with large-scale motion is relatively higher than that of fine-scales. The peak of the spectral density is preserved with depth, while the variance at all scales decreases with depth. The comparison between the two simulations is illustrated better in Figure 5a where we present the depth-averages of annual KE spectral density for the two simulations in the same region (Box 8). The spectral densities from the two models agree well with an approximate slope of -3 , a value that is characteristic for Quasi-Geostrophic (QG) prediction. In this QG regime, submesoscale structures are expected to be weakly energetic while energy is concentrated at the mesoscales. The depth-averages of the winter and summer KE spectral density are presented in Figure 5b. There is a seasonality in the spectral density that is mostly associated with an increase in the variance at submesoscales in winter. The spectral shape in both winter and summer are somewhat QG. We find this surprising given that previous studies (Callies \& Ferrari, 2013; Shcherbina et al., 2013; Sasaki et al., 2014) have shown that in the presence of intense submesoscales in winter, KE spectral density is likely to have $k^{-2}$ or $k^{-5 / 3}$ spectral shape.

As previously highlighted, daily averaging the velocity fields before spectral estimation could suppress the signature of high-frequency motions (unbalanced submesoscales, ageostrophic wave motions) and this could affect the distribution of energy implied from this sort of spectral analysis. To ascertain this, we compared NATL60 surface KE spectral density for hourly averages versus daily averages in Figure 6a. This comparison is only possible for NATL60 because HYCOM50 dataset is stored in daily averages except for the last month of the simulation. In this comparison for NATL60 (Figure 6a), submesoscale motions are more energetic than presented in the daily spectral density. There is a tendency for the distribution (annual average) of energy at submesoscales in the hourly spectral to follow a $k^{-2}$ spectra shape, a characteristics of a regime associated with fronts. 
The winter versus summer spectral density computed from NATL60 hourly averages of the velocity field (Figure 6b), clearly show how high-frequency motions and energetic submesoscales drive the seasonality of kinetic energy distribution at fine-scales. There is a shift in the spectral slope from -3 in summer to -2 in winter. This shift can be interpreted as a change in dynamics from the interior QG Philips-like regime to a surface intensified Charney-like regime (Sasaki et al., 2014). In this Charney-like regime, submesoscales are associated with large vertical velocity and, in turn, large submesoscale buoyancy fluxes that are suggested to feed mesoscales through an inverse kinetic energy cascade. This result is consistent with the findings of Sasaki et al. (2014); Callies and Ferrari (2013), and it further evaluates the ability of NATL60 to resolve physical processes at fine-scales. In section (4), we shall also discuss how this regime change from summer to winter affects the redistribution of kinetic energy.

\subsection{Spectral Slope}

As we have shown in the previous section, a quick way to estimate the wavenumber spectral power law is to compute the 1D wavenumber spectral density then estimate a slope from this spectral by fitting a line to the spectral density curve within a selected wavenumber range. This method is fast and easy to implement and provides a way to investigate regional variability of ocean energetics both at the basin and global scale. For studies on mesoscale energetics using satellite datasets and model outputs, this wavenumber range is mostly within the error limits of the altimeter instrument $(\sim 70 \mathrm{~km})$ and the horizontal scale of meso/large scale motion (250-300 km). One drawback of this approach is that it does not account for the changes in the scale of average energetic eddy structures with latitude. Scales of motions that are mesoscales in the polar regions could be classified as submesoscales in the tropics.

To characterize the spectral signature correctly, several recent studies have tried to propose different approaches to estimate the wavenumber spectral power law. For mesoscale resolving altimetry datasets, Vergara et al. (2019) estimated spectral slope between the peak of the spectral and the minimum of the Rossby radius and the Rhines scale following Eden (2007). A similar approach was presented in Sasaki and Klein (2012), where the authors estimated spectral slope between a fixed wavelength of $30 \mathrm{~km}$ (at the lower bound), and a scale that corresponds to the peak of the KE wavenumber spectral.

In order to show how sensitive the estimated slopes are to the selected wavelength range, we present in Figure 7a the average KE wavenumber spectral density and slope for box 3 in March for three different selected wavenumber ranges. The dashed lines with colors red, blue, and black represent the 10-100 km, 10-250 km, and 70-250 km, wavelength, 
respectively. The estimated slopes for these three different wavelength ranges have different values, therefore raising the question as to which slope is most representative of the dynamics of this region. We repeat this analysis for all the boxes and present the map in Figure 8. The mismatch is particularly pronounced in the sub-polar region, where the scales of the eddy structures are relatively smaller. The $70-250 \mathrm{~km}$ wavelength range is a typical wavelength for estimating spectral slope for satellite datasets because $70 \mathrm{~km}$ roughly corresponds to the wavelength where the satellite data becomes noisy. The spectral slope in this range is consistent with the already published work of Dufau et al. (2016) and Chassignet and Xu (2017).

To avoid the sensitivity of the estimated spectral slope to an a-priori selected wavelength range, we introduce an approach that takes into account the dynamics of the regions and the resolving capability of the model by estimating the spectral slope (Figure 7b) between the energy-containing scale (Kjellsson \& Zanna, 2017) and the effective resolution of the model (Soufflet et al., 2016). The energy-containing scale (which represents the scale of the most energetic eddy structure) is estimated from the kinetic energy wavenumber spectral using equation (3) while the effective resolution (a function of the model grid-size) is taken as $5 \times$ the model grid size, which is roughly equal to $10 \mathrm{~km}$ for both models. This approach takes into account the scale of the energetic eddy structures within the flow region and also takes into account the geographical variability of this scale, and therefore provides a way to infer dynamical properties of oceanic motions in different regions.

$$
\lambda_{e}=\frac{\iint E\left(k_{x}, k_{y}\right) d k_{x} d k_{y}}{\iint \sqrt{k_{x}^{2}+k_{y}^{2}} E\left(k_{x}, k_{y}\right) d k_{x} d k_{y}}
$$

We apply this technique to the output of both simulations (KE spectral density from daily averages), and we present the estimated spectral slope and the energy-containing scale (integral scale) for all the boxes in the North Atlantic (Figure 9). The estimated integral scale from the wavenumber spectral density represents the averaged scale of energetic structures in the selected region. On one hand, this scale varies regionally and fairly follows the variability of the Rossby radius of deformation with latitude, with high values in the south and relatively low values in the north. On the other hand, the estimated slope across the basin is almost uniform and follows the prediction of QG with a slope value $\sim k^{-3}$. This consistency with the QG prediction is observed in both model outputs and also holds in the well known high energetic Gulf stream (box 1) and the low energetic OSMOSIS (box 10) regions. This result form daily fields comes with the caveat of suppressing the impact of high-frequency motions on the estimated slope values. 
In order to investigate whether accounting for high-frequency motions would affect our estimation of spectral slopes, we estimate the spectral slope (from KE spectral density computed using hourly averages of velocity fields). We do this only for NATL60 because HYCOM50 surface hourly output is available only for one month. The NATL60 spectral slope from hourly spectral density in all the regions (Figure 10) has smaller values than that of daily averages. As previously highlighted in section 3.1, this implies that the actual spectral are shallower in hourly fields (as a result of stronger variance at the fine-scales) compared to daily fields. The impact of intense submesoscale and ageostrophic flows is better illustrated in the winter/summer map of the spectral slope (Figure 11). The slopes in winter have values that are closer to $k^{-2}$ throughout the domain. This indicates that high-frequency motions are quite significant in the North Atlantic ocean basin. In the next section, we shall discuss the impact of different dynamics on the redistribution of kinetic energy.

\section{Kinetic Energy Cascade}

In this section, we present and discuss the exchange of energy due to non-linearity across different scales of motion. This exchange is estimated from the horizontal velocity fields using equation (2). A positive flux represents a direct cascade of energy, while a negative value represents an inverse cascade of energy.

\subsection{Annual Averages of Kinetic Energy Cascade}

We show in Figure 12 the KE spectral flux computed using one year's daily outputs of surface velocity fields. For simplicity, we show plots for boxes 3, 8, and 11 representing latitudes of $35^{\circ} \mathrm{N}, 45^{\circ} \mathrm{N}$, and $55^{\circ} \mathrm{N}$, respectively. In all the boxes and both models, the spectral flux is dominated by an inverse cascade of energy at large scales (between 25-50 $\mathrm{km}$ and $500 \mathrm{~km}$ ) and a forward cascade of energy below 25-50 km. As observed earlier, the spectral slope from daily averaged fields has a value that is $\sim-3$, a characteristic of QG turbulence. The energy exchanges computed using the same data show that the flux is mostly upscale with a little forward flux at fine-scales. While the inverse cascade is a well known phenomenon in geostrophic turbulence, the dynamics responsible for the forward cascade at fine-scales is, however, still a subject for discussion. One would expect a forward flux at fine-scales to be accompanied by a $\left(k^{-2}\right.$ or $\left.k^{-5 / 3}\right)$ spectral slope. This sort of relationship between spectral density and flux is not observed for estimates coming from the daily fields. This finding is, however not new and agrees with the results of Brüggemann and Eden (2015). The authors show that as soon as ageostrophic dynamics become important in a quasi-QG flow, the flow is no longer restricted to an inverse cascade of energy. Instead, 
the kinetic energy can proceed toward smaller scales providing a direct route to dissipation. We believe the fine-scale ageostrophic motions resolved by these simulations are significant for the flow to be in a regime that supports a forward cascade of energy.

It is interesting to note that the scale at which the inverse cascade is most intense coincides with the energy-containing scale (dashed line in Figure 12 estimated from the kinetic energy spectral density). This signifies that the inverse cascade is maximum at the scale of the most energetic eddies. Just like the energy-containing scale, the scale of the most intense inverse cascade varies with latitude with relatively smaller values in the sub-polar regions (Figure 13). Also, depending on the region, part of the submesoscale range (0 to 25$50 \mathrm{~km}$ ) falls to the left of the zero-crossing (where the flux changes sign). This implies that submesoscales motions are involved in fluxing energy to large-scale via an inverse cascade of energy. This scale of zero-crossing varies across the basin with higher values in high EKE regions and relatively smaller values in low EKE regions (Figure 13). This regional variability in the value of the zero-crossing shows that at fine-scales, the spatial scale at which energy is injected into the flow is greater in eddy-rich regions, compared to eddy-poor regions.

Figure 14 presents the KE spectral flux for Box 8 as a function of depth. The overall shape of the flux is preserved, and the scale at which the inverse cascade is maximum is also consistent with depth. The strength of the inverse cascade decreases with depth, and the direct cascade at high wavenumbers is confined mostly to the surface. In section 3.1, the question was raised as to whether HYCOM50's higher KE, when compared to NATL60, is a consequence of HYCOM50's coarser vertical resolution, which could lead to a surface intensified inverse cascade and hence more energetic surface eddies. However, in Figure 14, we can see that both at the surface and all depth levels, the estimated inverse and direct cascade is stronger in HYCOM50 than in NATL60. This indicates that HYCOM50 upscale energy flux is not surfaced intensified and we can conclude that the disparity between the two models in terms of energy levels is most likely due to differences in the length of the spin-up as well as the choice of sub-grid scale parametrization, and that having only 32 isopycnal vertical levels is not detrimental to the representation of the dynamics in the ocean interior.

\subsection{Seasonality of Energy Cascade}

In this section, we present the seasonality of the kinetic energy spectral flux by comparing winter (JFM) and summer (JAS) averages. Figure 15 shows the winter cascade (in blue) and the summer cascade (in red). There are two notable differences between the seasons. First, there is a shift in the zero crossings to higher wavenumbers in winter. Second, there 
is a stronger forward cascade within the submesoscale range in winter. As highlighted in the preceding section, a zero-crossing at the high wavenumbers partly indicates how much submesoscale motions are involved in feeding large-scale motions via an inverse cascade of energy. So, a shift to higher wavenumbers in wintertime signifies that smaller-scale structures are involved in fluxing energy to larger scales. It is noteworthy that the integral scale and scale of the maximum inverse cascade also undergo seasonality. There is a shift in the scale to high wavenumber from winter to summer. This can be interpreted as a reduction in the average size of energetic eddies structures in winter. This sort of seasonality in eddy length-scale is documented in Ajayi et al. (2020).

It is interesting to understand the contribution of the different dynamics on the seasonality of the energy cascade. Recent studies have shown that submesoscales are energetic in wintertime (Mensa et al., 2013; Sasaki et al., 2014; Callies, Flierl, et al., 2015; Rocha et al., 2016), and their emergence is forced by mechanisms such as frontogenesis, wind-induced frontal instabilities, mixed layer instability among many others (Thomas, 2008; McWilliams, 2016). Sasaki et al. (2014) argued that submesoscales generated via mixed layer instability could feed large scale motion via an inverse cascade of energy, hence a shift in the zerocrossing towards high wavenumbers. More recently, Schubert et al. (2020) used both the coarse-graining approach (Aluie et al., 2017) and spectral analysis to investigate the role of mixed layer baroclinic instabilities on kinetic energy exchanges. Their results show that mesoscale oceanic eddies are strengthened by the absorption of submesoscale mixed layer eddies and that the forward cascade of energy at very fine-scales occur mostly in frontogentic regions. Following these aforementioned findings, we hypothesize that the increased forward cascade presented in this study, could be associated with frontogenesis and submesoscale frontal instabilities. This seasonality highlights how submesoscale motions modulate the redistribution of energy between scales of motions; hence, the need for climate (ocean) models with submesoscale resolving capability.

\subsection{Impact of High-Frequency Motions on Energy Cascade}

In section 3.1, we observed that in the presence of high-frequency motions, the spectral densities (computed from hourly fields) are shallower with an increased variance at finescales compared to daily averaged fields. In this section, we are going to discuss the impact of this increased variance on energy exchanges.

The contribution of high-frequency motions to kinetic energy spectral flux is highlighted in Figure 16a where we show the comparison of the spectral flux computed from daily versus hourly fields. The magnitude of the forward cascade at submesoscales is significantly stronger in hourly spectral flux. That high-frequency motions can provide a pathway to 
kinetic energy dissipation is illustrated in these results. The dynamics responsible for this increase in forward cascade are likely due to an energy loss to the generation of gravity waves and unbalanced submesoscales. It is interesting to note that the scale of the most intense inverse cascade remains the same while there is a slight shift in the zero-crossing towards higher wavenumber. This result suggests that the impact of high-frequency motion on energy exchanges is mostly concentrated at fine-scales. As seen in the daily averages, the winter flux is equally stronger in hourly fields (Figure 16a). We believe that this increase is attributed to resolved internal gravity waves and intense (un)balanced submesoscales. However in summer time both daily and hourly flux are identical.

\subsection{Diagnosing Spectral Flux from SWOT}

NATL60 and HYCOM50 are submesoscale permitting model simulations that have been created to simulate the scales of motions that we expect SWOT to see from space. SWOT will provide measurements of sea surface heights from which velocities (based on geostrophic approximations) will be inferred. Geostrophically balanced motions dominate the ocean at meso/large-scale, and the inferred geostrophic velocities at this scale mostly reflect the absolute velocity of these large scales motions. However, geostrophy is less accurate for fine-scale motions, particularly at the submesoscales and this remains a challenge due to the projection of ageostrophic motions on SSH field. Recent studies have shown that some classes of non-wave ageostrophic motions could impact the forward cascade of kinetic energy at fine-scales (Capet, McWilliams, et al., 2008). We have equally shown in the previous sections that high-frequency motions in the form of ageostrophic waves and unbalanced submesoscales can contribute significantly to a forward cascade of energy at fine-scales. In light of this, we are not sure if SWOT data can accurately estimate the redistribution of kinetic energy at fine-scales, given that SWOT will provide information down to $\sim 15$ $\mathrm{km}$. We are curious to see if the geostrophically inferred surface velocity would capture the accurate energetics at scales $<50 \mathrm{~km}$ where geostrophy is likely to fail.

To investigate this, we present in Figure 17 the spectral flux from total velocity and geostrophic velocity for three regions (same as for the previous sections). The geostrophic velocity is estimated from the sea surface height (SSH) using the geostrophic approximation. The strength of the energy cascade differs between the flux computed from the total velocity and that of the geostrophic velocity. This difference is consistent in all three boxes and in the two models. In particular, at the very high wavenumbers, the forward cascade is underestimated in the flux computed from the geostrophic velocity. A possible reason for this mismatch at smaller scales could be explained by the findings of Brüggemann and Eden (2015) that showed that ageostrophic flows at fine scales are an excellent catalyst for energy 
cascade towards dissipation. Despite the differences in terms of flux magnitude, the overall shape of the flux is consistent for the two forms of spectral flux. The scale at which the inverse cascade is maximum is the same irrespective of the type of velocity fields.

\section{Discussion and Summary}

In this study, we presented the analysis of kinetic energy wavenumber spectral density, slope, and flux by using datasets from daily and hourly outputs of two submesoscale permitting ocean models of the North Atlantic. The analysis presented has shown that in summer, the North Atlantic ocean follows the QG framework (with $\sim k^{-3}$ spectral shape) and in winter, the basin mostly reflects a $k^{-2}$ spectral shape, a characteristic of a front dominated regime. The estimated kinetic energy spectral flux revealed an overall net inverse cascade of energy with a significant direct cascade of energy at high wavenumbers. The spectral flux undergoes a seasonality that is associated with a stronger forward cascade at high wavenumbers in winter. This increased forward cascade in winter is further amplified in the presence of high-frequency motions. The spectral flux as a function of depth reveals that the forward cascade at high wavenumbers is confined to the mixed layer while the inverse cascade dominates the water column down to $700 \mathrm{~m}$. We showed that the maximum inverse cascade occurs at a scale that coincides with the energy-containing scale.

Until recently, most basin/global scale simulations had their outputs stored in the form of daily averages. Our results show that high-frequency motions (that are only resolved in hourly outputs) affect the distribution and exchanges of kinetic energy. We observed that the difference between the daily and hourly results is mostly in the form of an increased variance and (forward) cascade at fine-scales in favor of hourly fields. High-frequency motions are dominated mainly by ageostrophic motions that include unbalanced submesoscales and fast propagating internal gravity waves. These two classes of motions are out of phase seasonally with stronger submesoscales in winter and stronger internal gravity waves in summer. It is puzzling that while the exchanges of energy at fine-scale are unaffected by high-frequency motions in summertime, the distribution of energy shows the contrary. We observed higher variance at fine-scales in power spectral density estimated from hourly fields. The reason for this disparity between the impact of high-frequency on spectral density and flux in summertime is not apparent but would be an interesting subject to investigate further.

The kilometric simulations used in this study have similar horizontal grid spacing but different numerics, sub-grid parameterization, and vertical resolution. Despite these differences, the two simulations agree well on the overall dynamics of the North Atlantic. However, HYCOM50 is more energetic compared to NATL60 both at the surface and in the interior. We found the estimated cascade in HYCOM50 to be of higher magnitude 
compared to NATL60 for both direct and inverse cascade. The difference in energy levels between the two models could be due to the difference in length of spin-up or/and the choice of subgrid-scale parameterization. Initially, we thought that HYCOM50 having just 32 hybrid layers in the vertical, could lead to a more surface intensified energy cascade in HYCOM50 than in NATL60. Nevertheless, this is not the case, because across all scales and at depth, HYCOM50 seems to show stronger energetic compared to NATL60.

NATL60 and HYCOM50 are designed mainly to serve as an observational dataset for the anticipated SWOT mission. SWOT will provide sea surface height, and by using geostrophic approximation, we would obtain the geostrophic velocity, a requisite for computing crossscale energy transfer. At fine-scale, geostrophic approximation is however less accurate. Thus, accurately diagnosing surface velocity from sea surface height at fine-scales remains a challenge, and this has an impact on the estimate of kinetic energy cascade. Our results show that at fine-scales, not accounting for the ageostrophic motions could affect at all scales, the true magnitude of the estimated cascade of kinetic energy.

The results presented in this study are based on the output of ocean numerical simulations that are forced with realistic atmospheric winds. Recent literature suggests that air-sea coupling at fine-scales could affect the evolution and energetics of oceanic eddies. Renault et al. (2016) using a coupled/uncoupled model of the California Upwelling System argued that the ocean-atmosphere interactions have feedback that acts as an oceanic eddy killer. This feedback deflects energy from the geostrophic current into the atmosphere and dampens geostrophic kinetic energy. A possible future study would be to recompute the (kinetic energy transfer) diagnostics in this study using datasets from an ocean-atmosphere coupled simulation. This sort of analysis would take into account the direct impact of air-sea interaction on the ocean's kinetic energy exchanges.

\section{Acknowledgments}

We thank three anonymous reviewers for providing helpful and stimulating comments that have greatly improved the manuscript. Adekunle Ajayi is partly funded by the University Grenoble Alpes AGIR research grant. Aurlie Albert is funded by the CMEMS Global High-Resolution MFC 22-GLO-HR project. Julien Le Sommer contributions are funded by CNES through their participation in the SWOT Science Team. Eric Chassignet and Xiaobiao Xu are supported by the Office of Naval Research (Grant N00014-15-1-2594) and the NSF Physical Oceanography Program (Award 1537136). William Dewar is supported by the French "Make Our Planet Great Again" program managed by the Agence Nationale de la Recherche under the Programme dInvestissement dAvenir, with the reference ANR-18-MPGA-0002 and by NSF grants OCE-1829856 and OCE-1941963. We 
thank Laurent Brodeaux (Ocean-Next) for providing the comparison between NATL60 and SARAL AltiKa altimeter. HYCOM50 and NEMO-NATL60 data can be accessed at ftp://ftp.hycom.org/pub/xbxu/ATLb0.02/ and http://meom-group.github.io/swot-natl60/accessdata.html, respectively. 


\section{References}

Ajayi, A., Le Sommer, J., Chassignet, E., Molines, J., Xu, X., Albert, A., \& Cosme, E. (2020). Spatial and Temporal Variability of the North Atlantic Eddy Field From Two KilometricResolution Ocean Models. J. Geophys. Res. Ocean., 125(5). doi: $10.1029 / 2019 \mathrm{jc} 015827$

Aluie, H., Hecht, M., \& Vallis, G. K. (2017). Mapping the Energy Cascade in the North Atlantic Ocean: The Coarse-graining Approach. J. Phys. Oceanogr., 225-244. doi: 10.1175/JPO-D-17-0100.1

Amores, A., Jorda, G., Arsouze, T., \& Le Sommer, J. (2018). Up to What Extent Can We Characterize Ocean Eddies Using Present-Day Gridded Altimetric Products? Journal of Geophysical Research: Oceans, 123. doi: 10.1029/2018JC014140

Barkan, R., Winters, K., \& McWilliams, J. C. (2017). Stimulated imbalance and the enhancement of eddy kinetic energy dissipation by internal waves. Journal of Physical Oceanography, 47(1), 181198. doi: https://doi.org/10.1175/JPO-D-16-0117.1.

Brannigan, L., Marshall, D. P., Naveira-Garabato, A., \& George Nurser, A. J. (2015). The seasonal cycle of submesoscale flows. Ocean Modelling. doi: 10.1016/j.ocemod.2015 .05 .002

Brüggemann, N., \& Eden, C. (2015). Routes to Dissipation under Different Dynamical Conditions. J. Phys. Oceanogr., 45(8), 2149-2168. doi: 10.1175/JPO-D-14-0205.1

Buckingham, C. E., Lucas, N., Belcher, S., Rippeth, T., Grant, A., Le Sommer, J., ... Alberto, N. (2019). The contribution of surface and submesoscale processes to turbulence in the open ocean surface boundary layer. Journal of Advances in Modeling Earth Systems, 11. doi: https://doi.org/10.1029/2019MS001801

Callies, J., \& Ferrari, R. (2013). Interpreting Energy and Tracer Spectra of Upper-Ocean Turbulence in the Submesoscale Range (1200 km). J. Phys. Oceanogr., 43(11), 24562474. Retrieved from http://journals.ametsoc.org/doi/abs/10.1175/JPO-D-13 -063.1 doi: 10.1175/JPO-D-13-063.1

Callies, J., Ferrari, R., Klymak, J. M., \& Gula, J. (2015). Seasonality in submesoscale turbulence. Nature Communication, 6, 6862. doi: 10.1038/ncomms7862

Callies, J., Flierl, G., Ferrari, R., \& Fox-Kemper, B. (2015). The role of mixed-layer instabilities in submesoscale turbulence. J. Fluid Mech., 788, 5 - 41. doi: 10.1017/ jfm.2015.700

Capet, X., Campos, E. J., \& Paiva, A. M. (2008). Submesoscale activity over the Argentinian shelf. Geophysical Research Letters, 35(15), 2 - 6. doi: 10.1029/2008GL034736

Capet, X., McWilliams, J. C., Molemaker, M. J., \& Shchepetkin, A. F. (2008). Mesoscale to Submesoscale Transition in the California Current System. Part III: Energy Balance and Flux. J. Phys. Oceanogr., 38, 2256 - 2269. doi: 10.1175/2008JPO3810.1 
Charney, J. (1971). Geostrophic turbulence. Journal of Atmospheric Sciences, 28, 10871095.

Chassignet, E. P., \& Xu, X. (2017). Impact of Horizontal Resolution $\left(1 / 12^{\circ}\right.$ to $\left.1 / 50^{\circ}\right)$ on Gulf Stream Separation, Penetration, and Variability. Journal of Physical Oceanography, 47(8), 1999 - 2021. doi: 10.1175/JPO-D-17-0031.1

Ducousso, N., Le Sommer, J., Molines, J. M., \& Bell, M. (2017). Impact of the symmetric instability of the computational kind at mesoscale- and submesoscalepermitting resolutions. Ocean Modelling, 120(18 26). doi: https://doi.org/10.1016/ j.ocemod.2017.10.006

Dufau, C., Orsztynowicz, M., Dibarboure, G., Morrow, R., \& Le Traon, P. (2016). Mesoscale resolution capability of altimetry: Present and future. J. Geophys. Res. Oceans, 121, 1-18. doi: https://doi.org/10.1002/2015JC010904

Dussin, R., Barnier, B., Brodeau, L., \& Molines, J. M. (2018). The making of the DRAKKAR forcing set DFS5. Drakker. doi: https://doi.org/10.5281/zenodo .1209243

Eden, C. (2007). Eddy length scales in the North Atlantic Ocean. Journal of Geophysical Research, 112(C6), C06004. doi: 10.1029/2006JC003901

Ferrari, R., \& Wunsch, C. (2009). Ocean Circulation Kinetic Energy: Reservoirs, Sources, and Sinks. Annu. Rev. Fluid Mech., 41(1), 253-282. doi: 10.1146/annurev.fluid.40 .111406 .102139

Fresnay, S., Ponte, A. L., Le Gentil, S., \& Le Sommer, J. (2018). Reconstruction of the 3-D Dynamics From Surface Variables ina High-Resolution Simulation of North Atlantic. Journal of Geophysical Research: Oceans, 123. doi: 10.1002/2017JC013400

Fu, L. L., Chelton, D. B., Le Traon, P. Y., \& Morrow, R. (2010). Eddy Dynamics From Satellite Altimetry. Oceanography, 23(4), 14-25. doi: 10.5670/oceanog.2010.02

Khatri, H., Sukhatme, J., Kumar, A., \& Verma, M. K. (2018). Surface Ocean Enstrophy, Kinetic Energy Fluxes and Spectra from Satellite Altimetry. J. Geophys. Res. Ocean.. doi: 10.1029/2017JC013516

Kjellsson, J., \& Zanna, L. (2017). The Impact of Horizontal Resolution on Energy Transfers in Global Ocean Models. Fluids, 2(3), 45. doi: 10.3390/fluids2030045

Le Traon, P. Y., Klein, P., Hua, B. L., \& Dibarboure, G. (2008). Do altimeter data agree with interior or surface quasi- geostrophic theory? Journal of Physical Oceanography, 5(30), 11371142.

Le Traon, P. Y., Rouquet, M. C., \& Boissier, C. (1990). Spatial scales of mesoscale variability in the North Atlantic as deduced from Geosat data. Journal of Geophysical Research, 95, 20267. doi: 10.1029/JC095iC11p20267

McWilliams, J. C. (2016). Submesoscale currents in the ocean. Proc. R. Soc., A 472, 
20160117. doi: http://dx.doi.org/10.1098/rspa.2016.0117

Mensa, J. A., Garraffo, Z., Griffa, A., Ozgokmen, T. M., Haza, A., \& Veneziani, M. (2013). Seasonality of the submesoscale dynamics in the Gulf Stream region. Ocean Dynamics, 63, 923 - 941.

Qiu, B., Chen, S., Klein, P., Sasaki, H., \& Sasai, Y. (2014). Seasonal Mesoscale and Submesoscale Eddy Variability along the North Pacific Subtropical Countercurrent. Journal of Physical Oceanography, 44(12), 3079 - 3098. doi: 10.1175/JPO-D-14-0071 .1

Renault, L., Molemaker, M. J., McWilliams, J. C., Shchepetkin, A. F., Lemarié, F., Chelton, D., ... Hall, A. (2016). Modulation of wind work by oceanic current interaction with the atmosphere. J. Phys. Oceanogr., 46(6), 1685-1704. doi: 10.1175/JPO-D-15-0232 .1

Rocha, C. B., Gille, S. T., Chereskin, T. K., \& Menemenlis, D. (2016). Seasonality of submesoscale dynamics in the Kuroshio Extension. Geophysical Research Letters, 43, 11304 - 11311. doi: 10.1002/2016GL071349

Rocha, C. B., Wagner, G. L., \& Young, W. R. (2018). Stimulated generation: Extraction of energy from balanced flow by near-inertial waves. J. Fluid Mech., 847, 417-451.

Salmon, R. (1980). Baroclinic instability and geostrophic turbulence. Geophys. Astrophys. Fluid Dyn., 15, 167211.

Sasaki, H., \& Klein, P. (2012). SSH Wavenumber Spectra in the North Pacific from a High-Resolution Realistic Simulation. J. Phys. Oceanogr., 42(7), 1233-1241. doi: 10.1175/JPO-D-11-0180.1

Sasaki, H., Klein, P., Qiu, B., \& Sasai, Y. (2014). Impact of oceanic scale- interactions on the seasonal modulation of ocean dynamics by the atmosphere. Nature Communication, 5, 5636. doi: 10.1038/ncomms6636

Sasaki, H., Klein, P., Sasai, Y., \& Qiu, B. (2017). Regionality and seasonality of submesoscale and mesoscale turbulence in the North Pacific Ocean. Ocean Dynamics, 67, 1195 - 1216. doi: 10.1007/s10236-017-1083-y

Schlösser, F., \& Eden, C. (2007). Diagnosing the energy cascade in a model of the North Atlantic. Geophys. Res. Lett., 34(2), 1-5. doi: 10.1029/2006GL027813

Schubert, R., Gula, J., Greatbatch, R., Baschek, B., \& Biastoch, A. (2020). The Submesoscale Kinetic Energy Cascade: Mesoscale Absorption of Submesoscale MixedLayer Eddies and Frontal Downscale Fluxes. J. Phy. Ocean., 50(9), 25732589. doi: https://doi.org/10.1175/JPO-D-19-0311.1

Scott, R. B., \& Arbic, B. K. (2007). Spectral Energy Fluxes in Geostrophic Turbulence: Implications for Ocean Energetics. J. Phys. Oceanogr., 37(3), 673-688. doi: 10.1175/ JPO3027.1 
Scott, R. B., \& Wang, F. (2005). Direct Evidence of an Oceanic Inverse Kinetic Energy Cascade from Satellite Altimetry. J. Phys. Oceanogr., 35, 1650-1666. doi: 10.1175/ JPO2771.1

Shcherbina, A. Y., D’Asaro, E. A., Lee, C. M., Klymak, J. M., Molemaker, M. J., \& McWilliams, J. C. (2013). Statistics of vertical vorticity, divergence, and strain in a developed submesoscale turbulence field. Geophys. Res. Lett., 40, 47064711. doi: $10.1002 / \operatorname{grl} .50919$

Smith, S., \& Vallis, G. (2002). The Scales and Equilibration of Mid Ocean Eddies: Forced Dissipative Flow. J. Phys. Oceanogr., 32(6), 1699-1720.

Soufflet, Y., Marchesiello, P., Lemari, F., Jouanno, J., Capet, X., Debreu, L., \& Benshila, R. (2016). On effective resolution in ocean models. Ocean Model., 98, 36-50. doi: 10.1016/j.ocemod.2015.12.004

Stammer, D., \& Böning, C. W. (1992). Mesoscale Variability in the Atlantic Ocean from Geosat Altimetry and WOCE High-Resolution Numerical Modeling (Vol. 22) (No. 7). doi: 10.1175/1520-0485(1992)022〈0732:MVITAO $\rangle 2.0 . C O ; 2$

Thomas, L. (2008). Submesoscale processes and dynamics. Geophysical Monograph Series, 177. doi: https://doi.org/10.1029/177GM04

Tulloch, R., John, M., \& Chris, H. (2011). Scales, Growth Rates, and Spectral Fluxes of Baroclinic Instability in the Ocean. J. Phys. Oceanogr., 41(6), 1057-1076. doi: 10.1175/2011JPO4404.1

Uchida, T., Abernathey, R., \& Smith, S. (2017). Seasonality of eddy kinetic energy in an eddy permitting global climate model. Ocean Modelling, 118, 41 - 58. doi: 10.1016/ j.ocemod.2017.08.006

Uppala, S. M., Kållberg, P. W., Simmons, A. J., Andrae, U., da Costa Bechtold, V., Fiorino, M., ... Woollen, J. (2005). The ERA-40 re-analysis. Q. J. R. Meteorol. Soc., 131 (612), 2961-3012. doi: 10.1256/qj.04.176

Vergara, O., Morrow, R., Pujol, M.-I., Gerald, D., \& Ubelmann, C. (2019). Revised Global Wave Number Spectra From Recent Altimeter Observations. Journal of Geophysical Research : Oceans. doi: https://doi.org/10.1029/2018JC014844 
Table 1. Table of model parameters for NATL60 and HYCOM50

\begin{tabular}{lll}
\hline & NATL60 & HYCOM50 \\
\hline Domain & $26.5 \mathrm{~N}-65 \mathrm{~N}$ & $28-80 \mathrm{~N}$ \\
Numerical Code & Nemo v.3.6 & HYCOM \\
Horizontal grid & $1 / 60: 0.9-1.6 \mathrm{~km}$ & $1 / 50: 1.1-2.2 \mathrm{~km}$ \\
Vertical coordinate & Z partial cells & Hybrid (Z \& isopycnal) \\
Integration period & 6 Months & 20 years \\
Vertical grid & 300 Levels : 1-50 m & 32 Layers \\
Boundary conditions & GLORYS2v3 & GDEM \\
Atmospheric forcing & DFS5.2 & ERA-40 \\
Horizontal Viscosity & Implicit in momentum advection & Laplacian \& Biharmonic \\
\hline
\end{tabular}




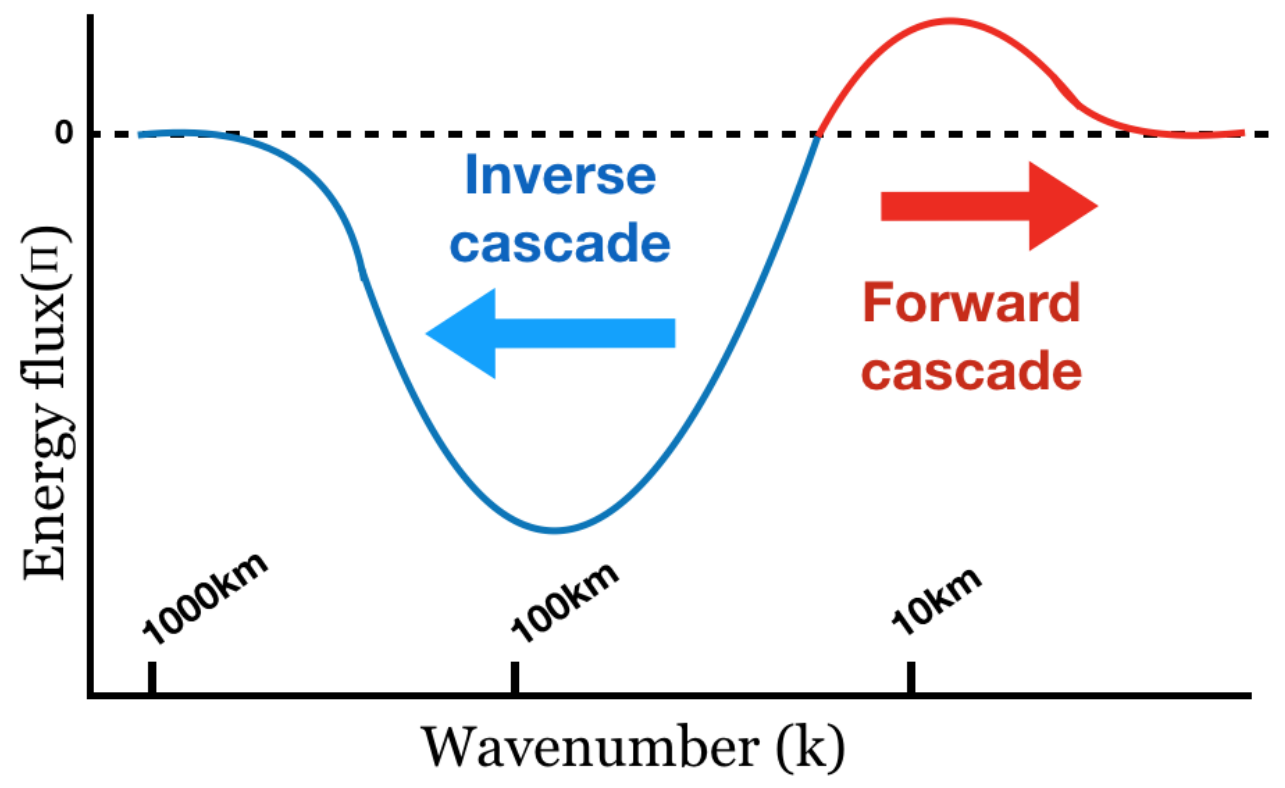

Figure 1. Schematics of kinetic energy spectral flux in the ocean at mid-latitude. Blue : inverse cascade of energy, Red : forward cascade of energy.

This article is protected by copyright. All rights reserved. 

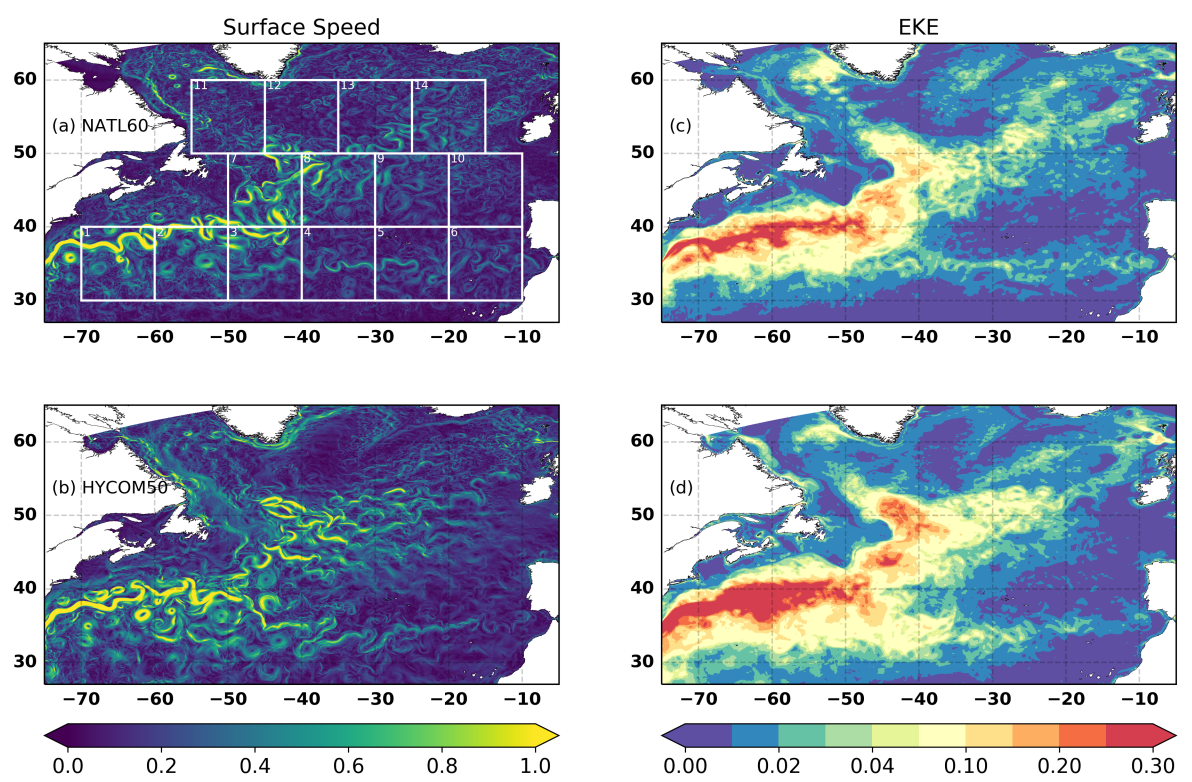

Figure 2. Left panel : snapshot of surface currents speed $(\mathrm{m} / \mathrm{s})$ on march 1st for NATL60 (a) and HYCOM50 (b). Right panel : surface eddy kinetic energy $\left(\mathrm{cm}^{2} \mathrm{~s}^{-2}\right)$ computed from daily output for NATL60 (c) and HYCOM50 (d). 


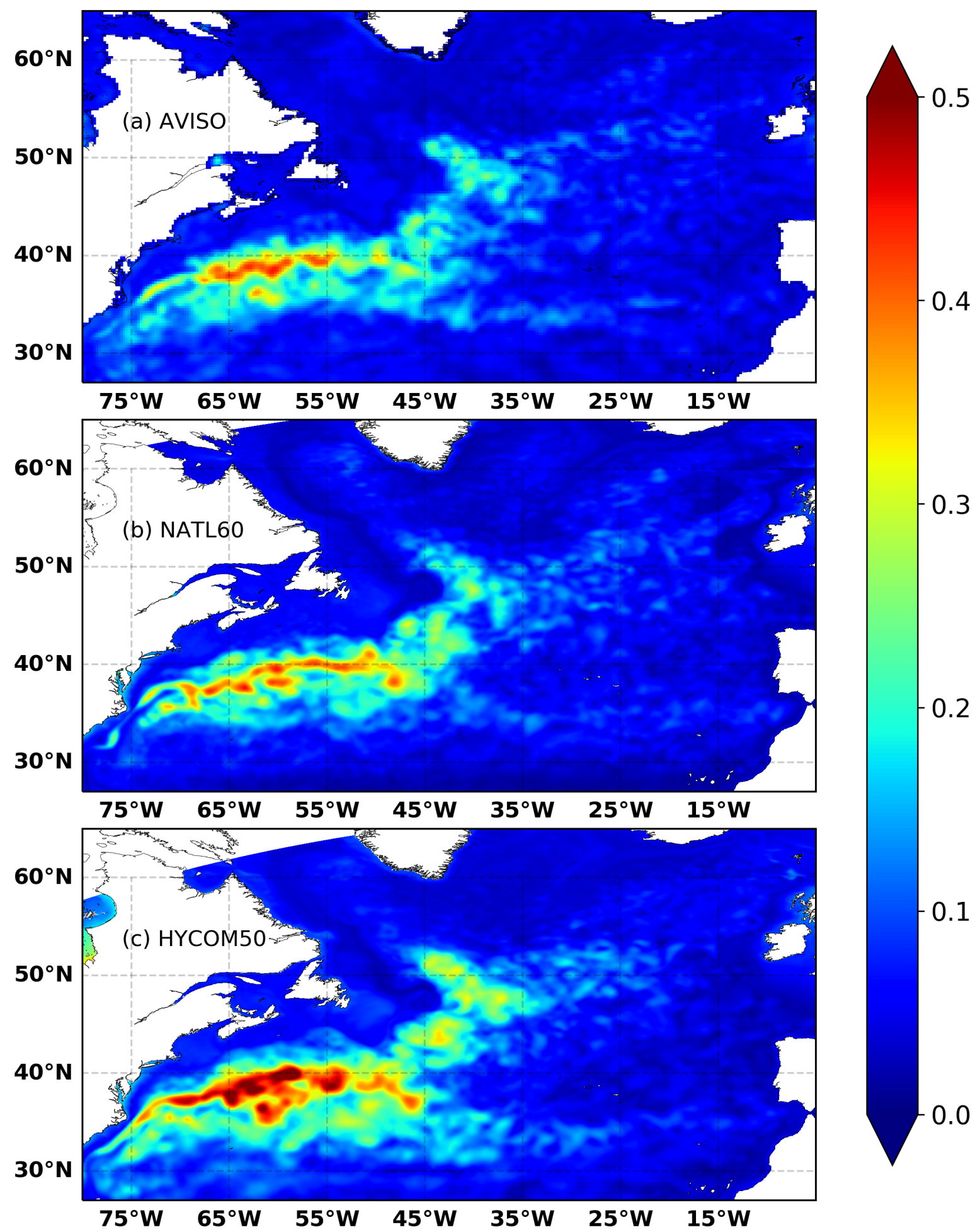

Figure 3. Standard deviation of sea surface height based on one year datasets for (a) AVISO,

(b) NATL60 and (c) HYCOM50. The SSH values for NATL60 and HYCOM50 were degraded to $0.25^{\circ}$ spatial resolution. In this comparison, we have used AVISO mean dynamical topography dataset from October 2012 to September 2013.

This article is protected by copyright. All rights reserved. 


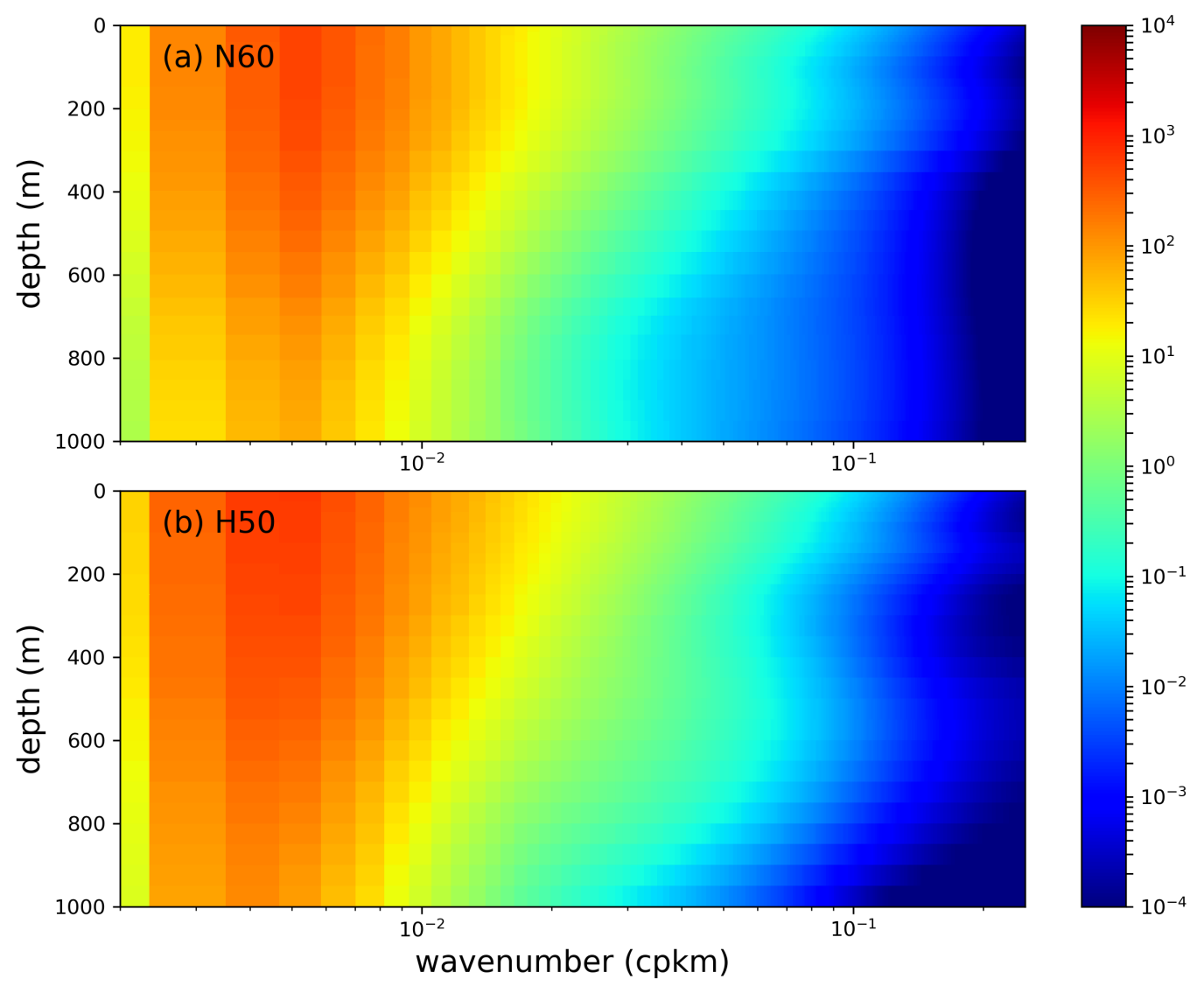

Figure 4. One year average of kinetic energy spectral density $\left(m^{2} s^{-2} / c p m\right)$ for Box 8 computed from horizontal total velocity as a function of depth for (a) NATL60 and (b) HYCOM50. 

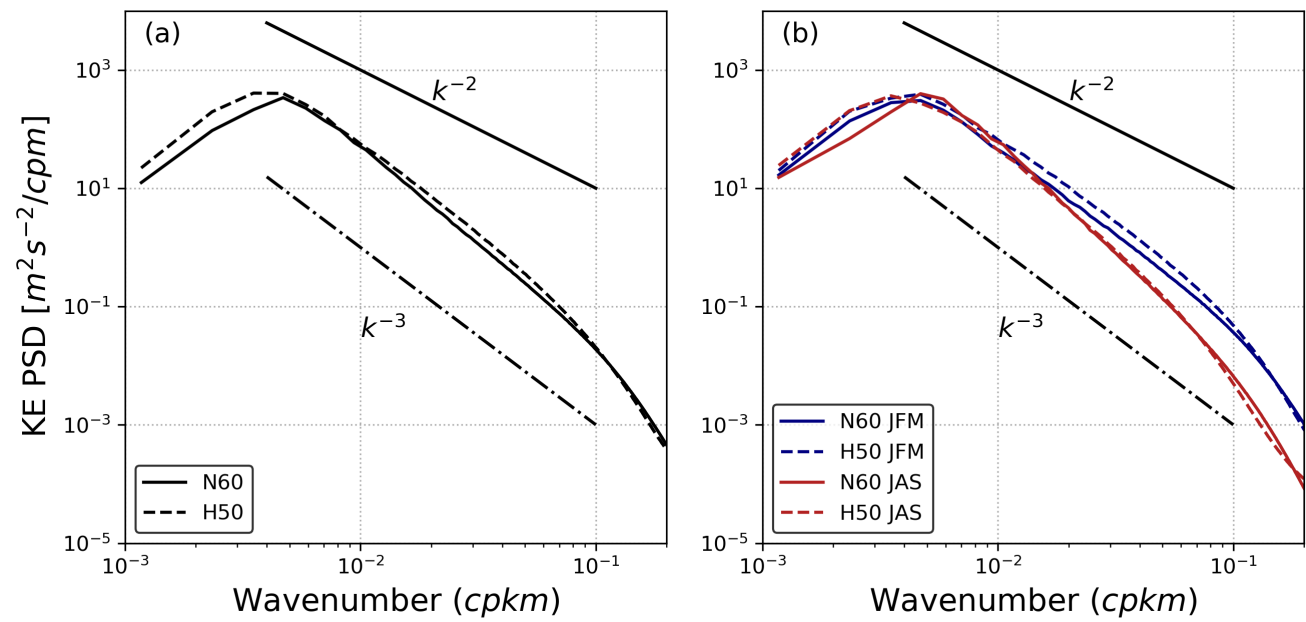

Figure 5. Kinetic energy spectral density for Box 8 (averaged over $1000 \mathrm{~m}$ depth) computed from daily output of horizontal total velocity for NATL60 (thick line) and HYCOM50 (dash line). (a) one year mean (b) winter (blue line) and summer (red line) averages. See Figure 5 in SI for a comparison of the surface vs depth averaged spectral density. A comparison of the spectral density between three different depth levels and the surface is presented in Figure 6 of SI 

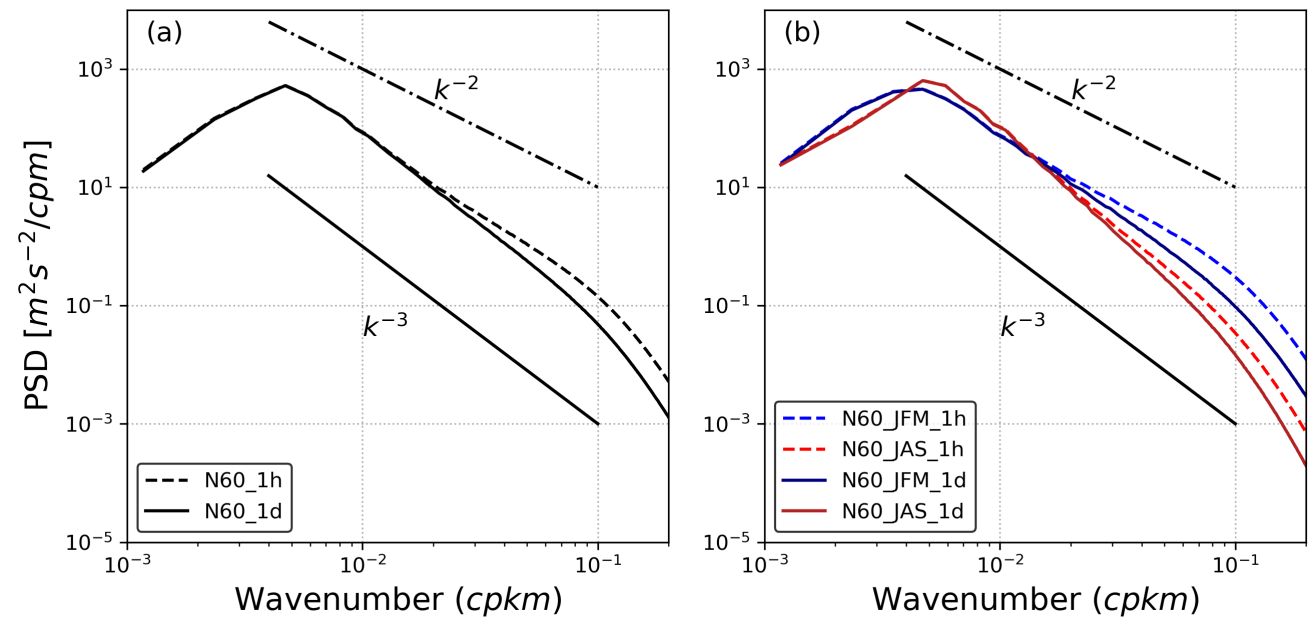

Figure 6. Comparison between surface kinetic energy spectral density computed from daily averages (thick line) and hourly averages (dash line) of velocity outputs for Box 8. (a) one year mean (b) winter (blue line) and summer (red line) averages. See Figure 7 and 8 in SI for a similar plots for all the boxes.) 

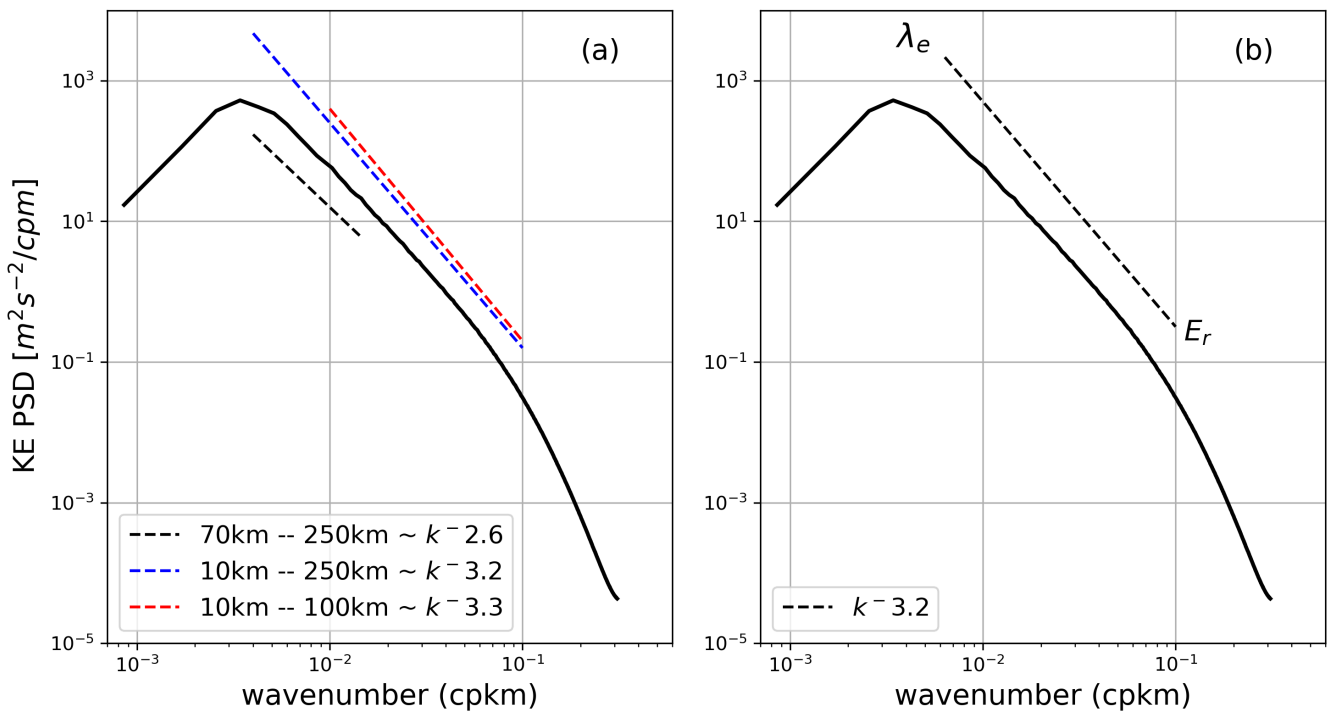

Figure 7. (a) Average surface KE spectral density and slope for box 3 (NATL60) in the month of March for three different selected wavenumber ranges. The wavelengths ranges are represented by dashed lines with the color red, blue and black for 10-100 km, 10-250 km and 70-250 km respectively. (b) A schematic to illustrate the proposed dynamical approach to estimate spectral slope. $\lambda_{e}$ is the energy-containing scale (which represents the scale of the most energetic eddy structure) and it is estimated from the kinetic energy wavenumber spectral density by using equation (3) while $E_{r}$ is the effective resolution (a function of the model grid-size) and is taken as $5 \times$ the model grid size. $E_{r}$ is roughly equally to $10 \mathrm{~km}$ for both NATL60 and HYCOM50. 

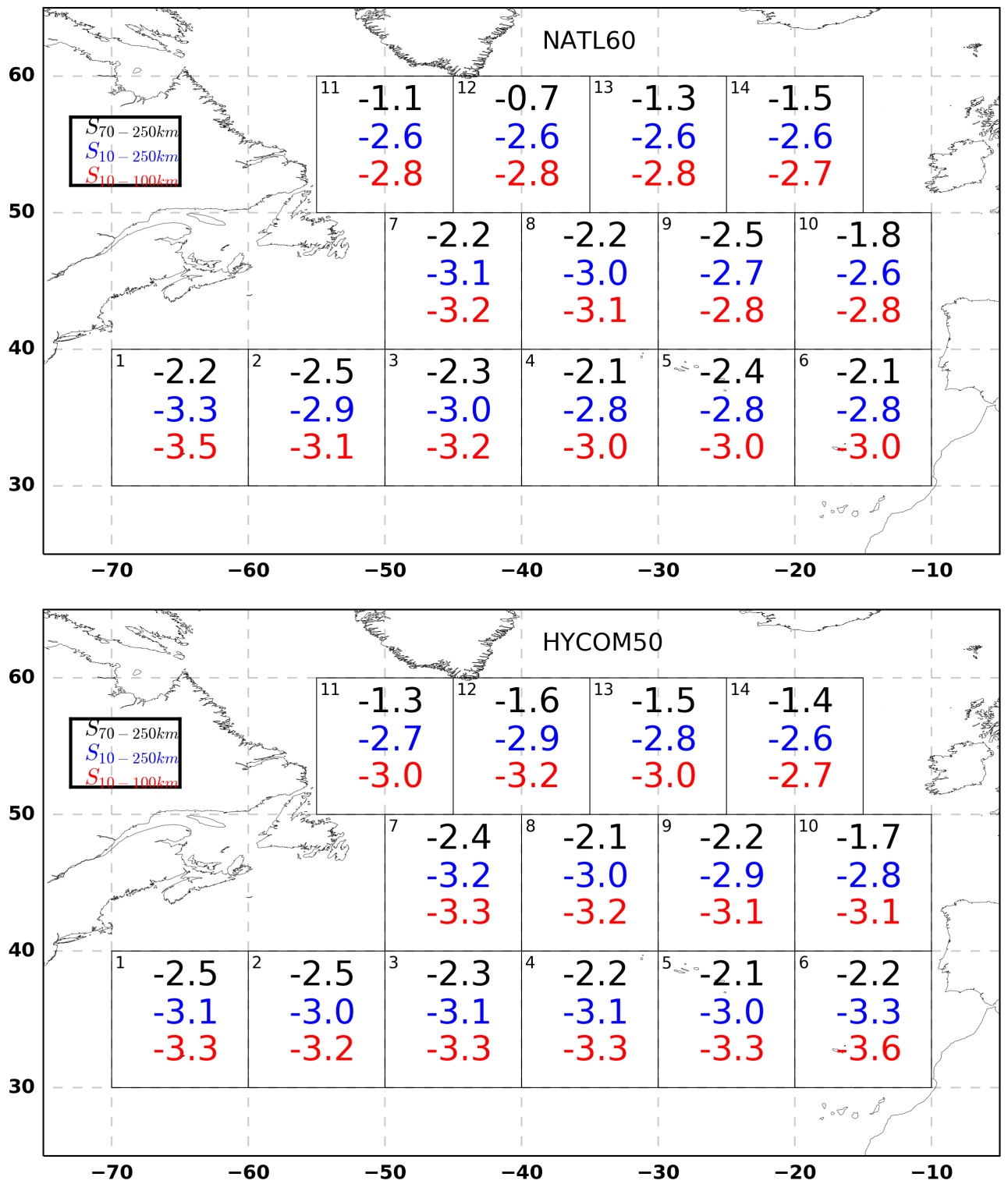

Figure 8. Map of spectral slope estimated from the surface kinetic energy spectral density for three different selected wavenumber ranges. Colour red, blue and black represent $10-100 \mathrm{~km}, 10$ - $250 \mathrm{~km}$ and $70-250 \mathrm{~km}$ respectively 

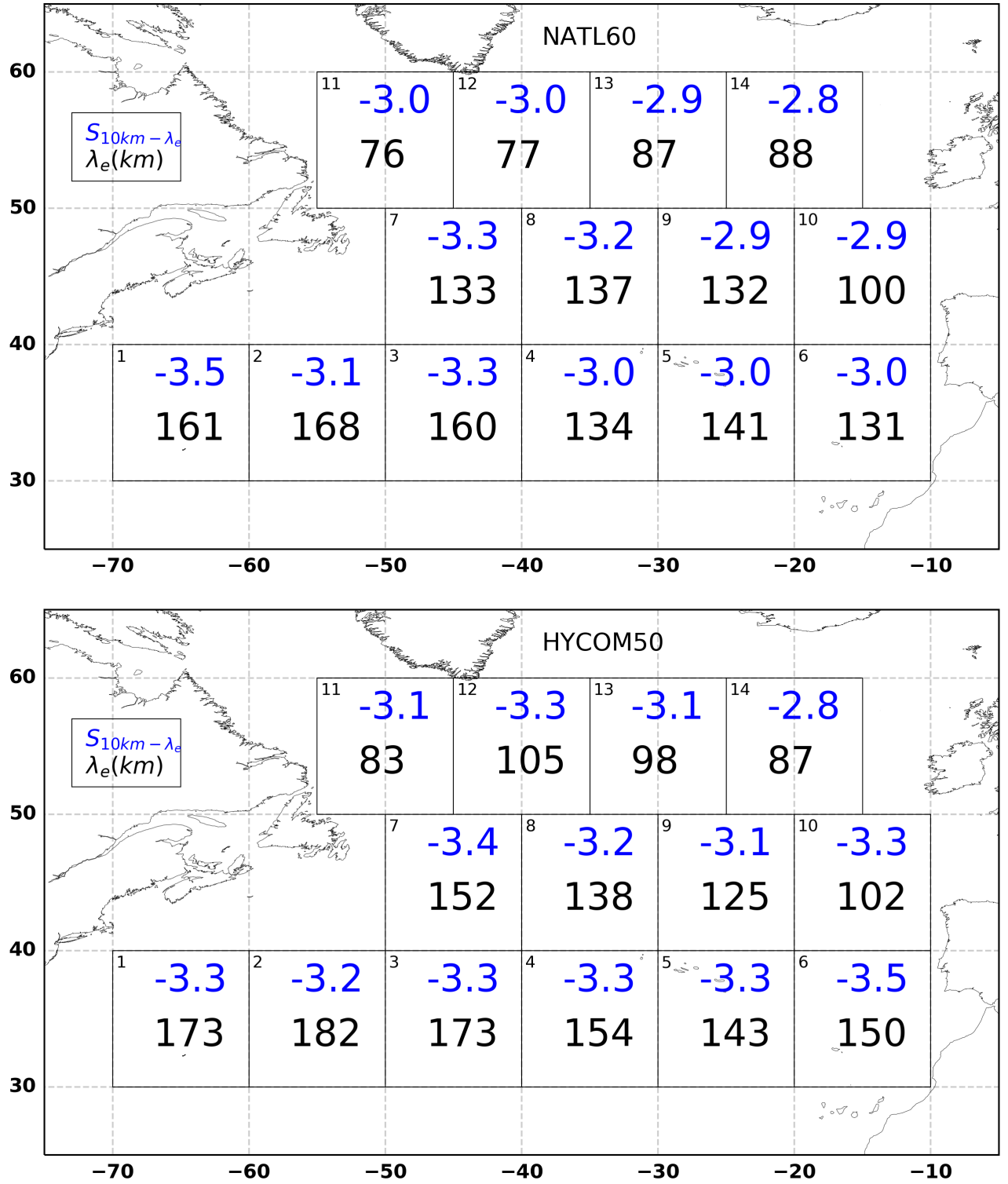

Figure 9. Map of spectral slope (blue colour) and energy containing scale, $\lambda_{e}$ (black colour) from the surface kinetic energy spectral density. The slope is estimated between the model effective resolution $\left(E_{f}\right)$ and the energy containing scale $\left(\lambda_{e}\right)$.

This article is protected by copyright. All rights reserved. 


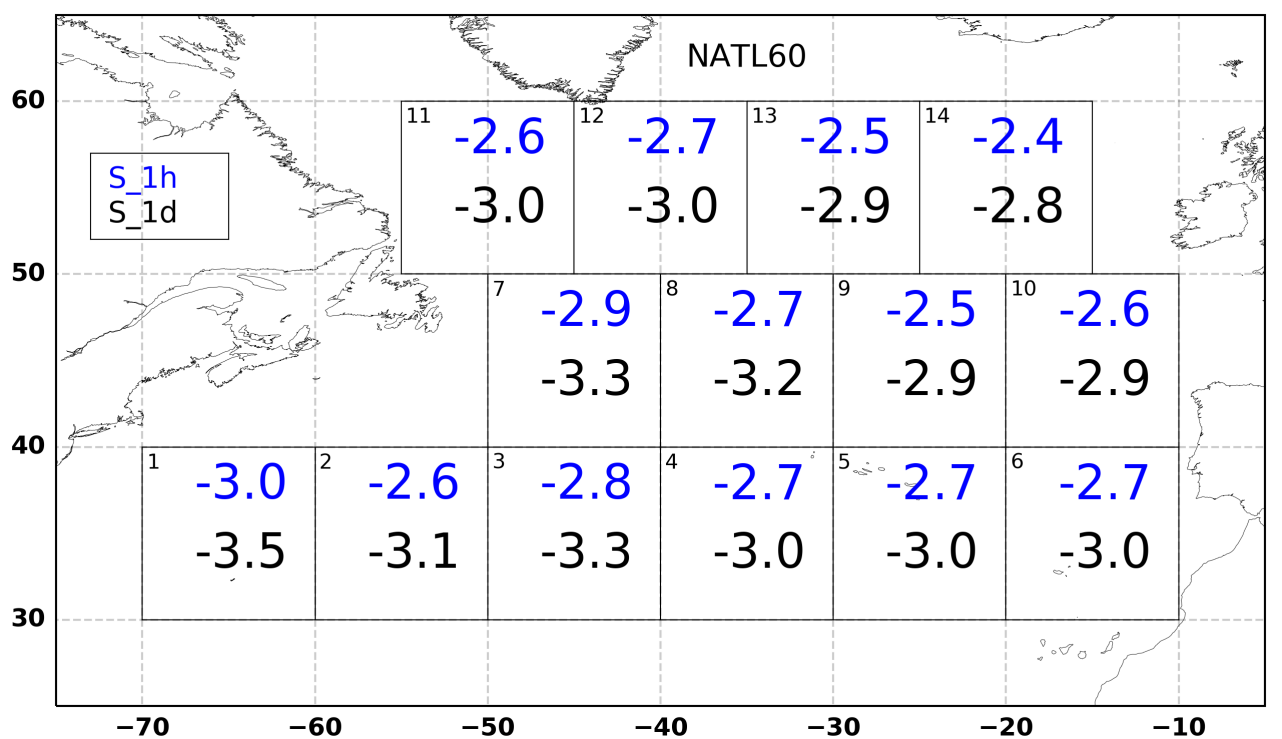

Figure 10. Map of spectral slope from the surface kinetic energy spectral density computed from daily versus hourly Fields. The slope is estimated between the model effective resolution $\left(E_{f}\right)$ and the energy containing scale $\left(\lambda_{e}\right)$.

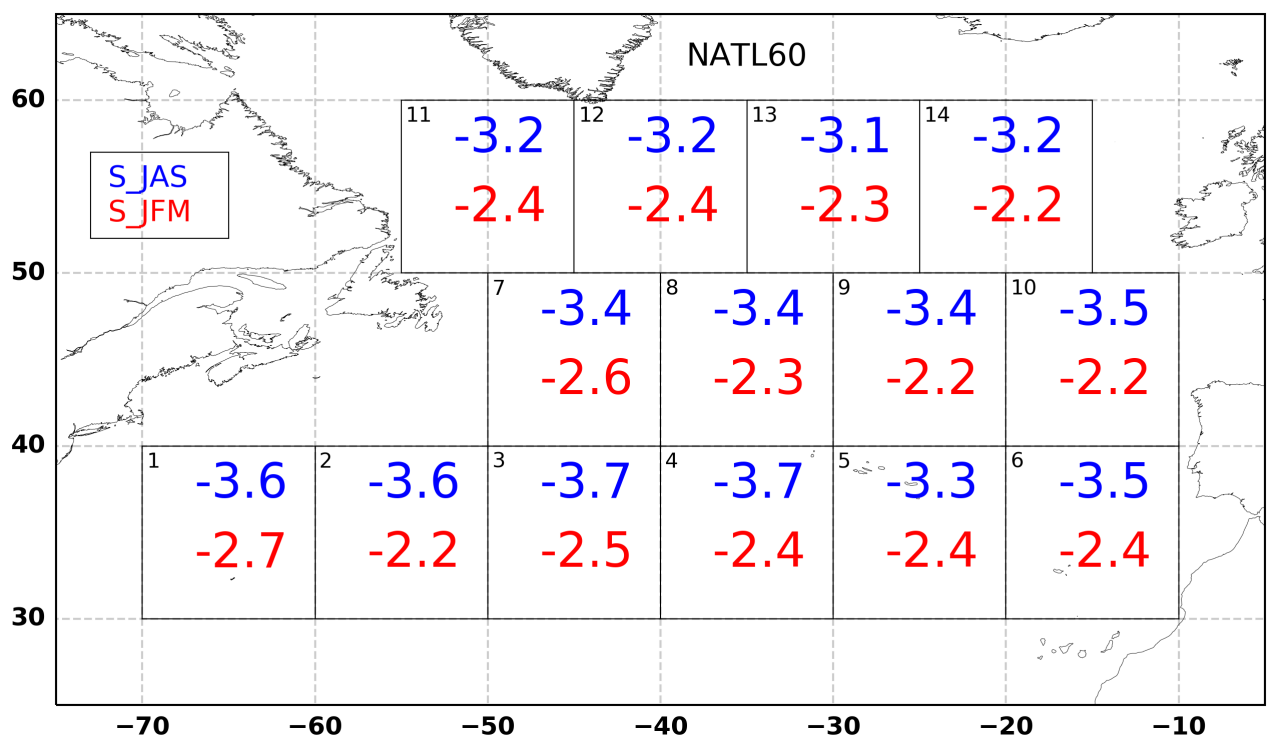

Figure 11. Map of spectral slope from NATL60 hourly surface kinetic energy spectral density in winter (JFM) and summer (JAS). The slope is estimated between the model effective resolution $\left(E_{f}\right)$ and the energy containing scale $\left(\lambda_{e}\right)$. 

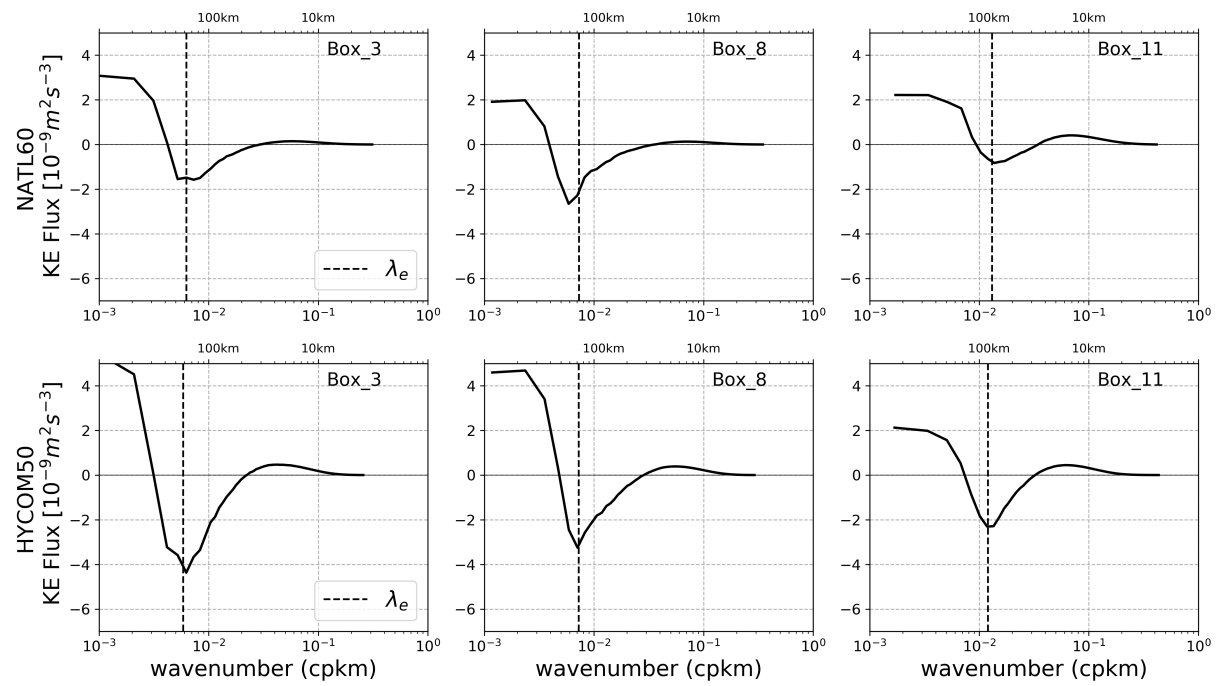

Figure 12. One year average of surface kinetic energy spectral flux computed from the daily output of horizontal total velocities. NATL60 (upper panel) and HYCOM50 (lower panel) 

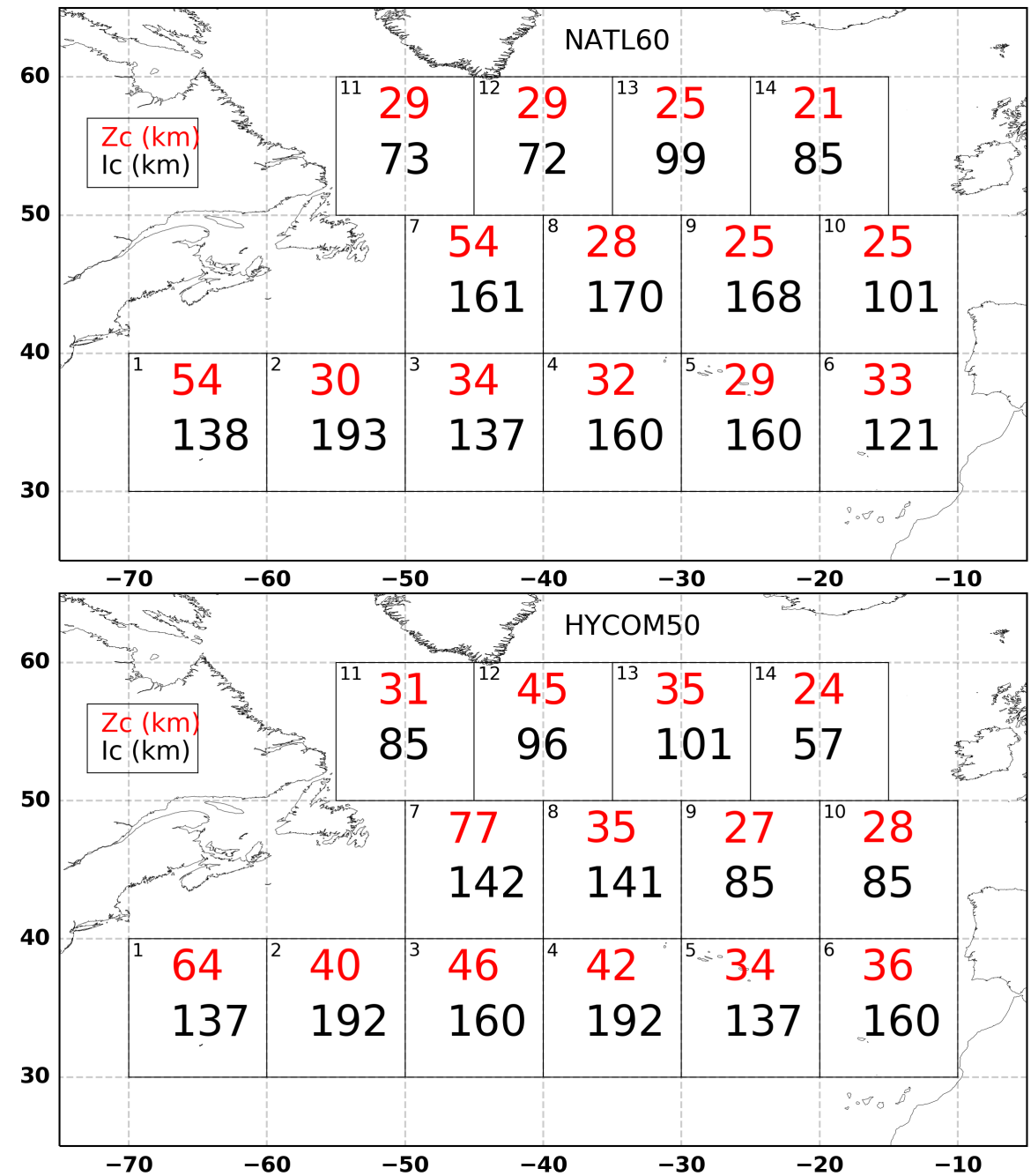

Figure 13. A geographical map of the scale of the kinetic energy spectral flux zero-crossing (red) and the most intense inverse cascade (black) estimated from one year average of the surface kinetic energy spectral flux for (a) NATL60 and (b) HYCOM50.

This article is protected by copyright. All rights reserved. 


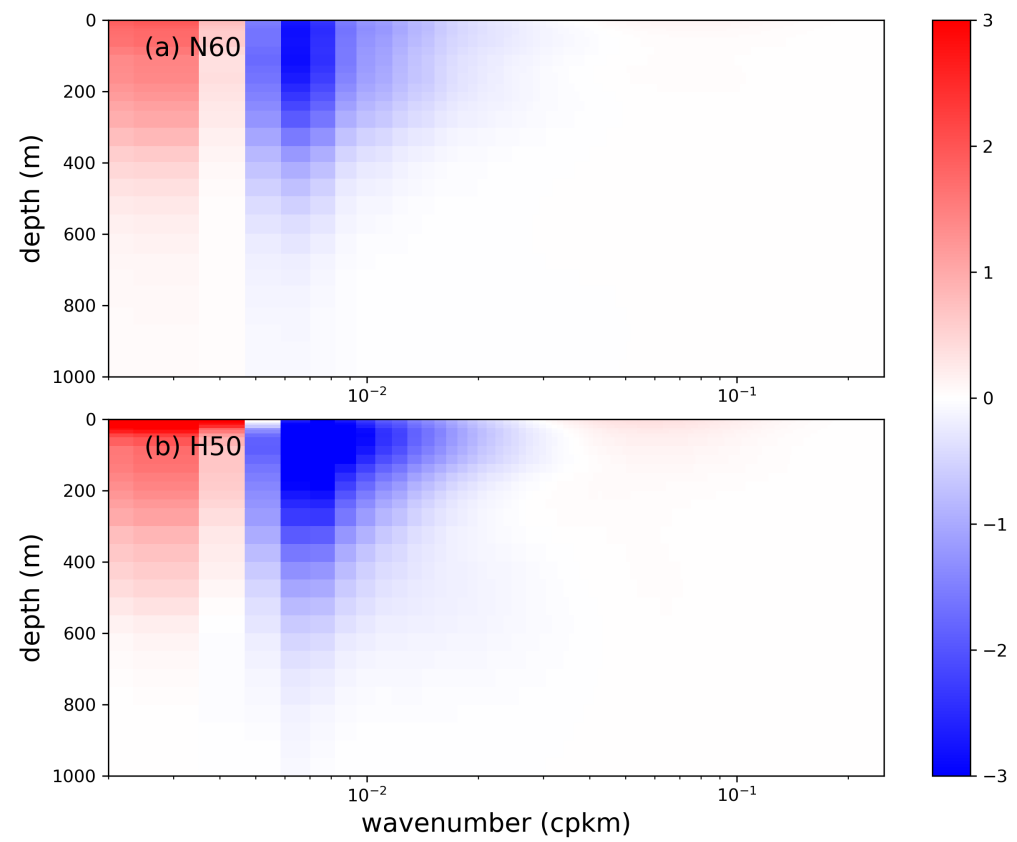

Figure 14. One year average of kinetic energy spectral flux for Box 8 computed from horizontal total velocity as a function of depth for (a) NATL60 and (b) HYCOM50. 

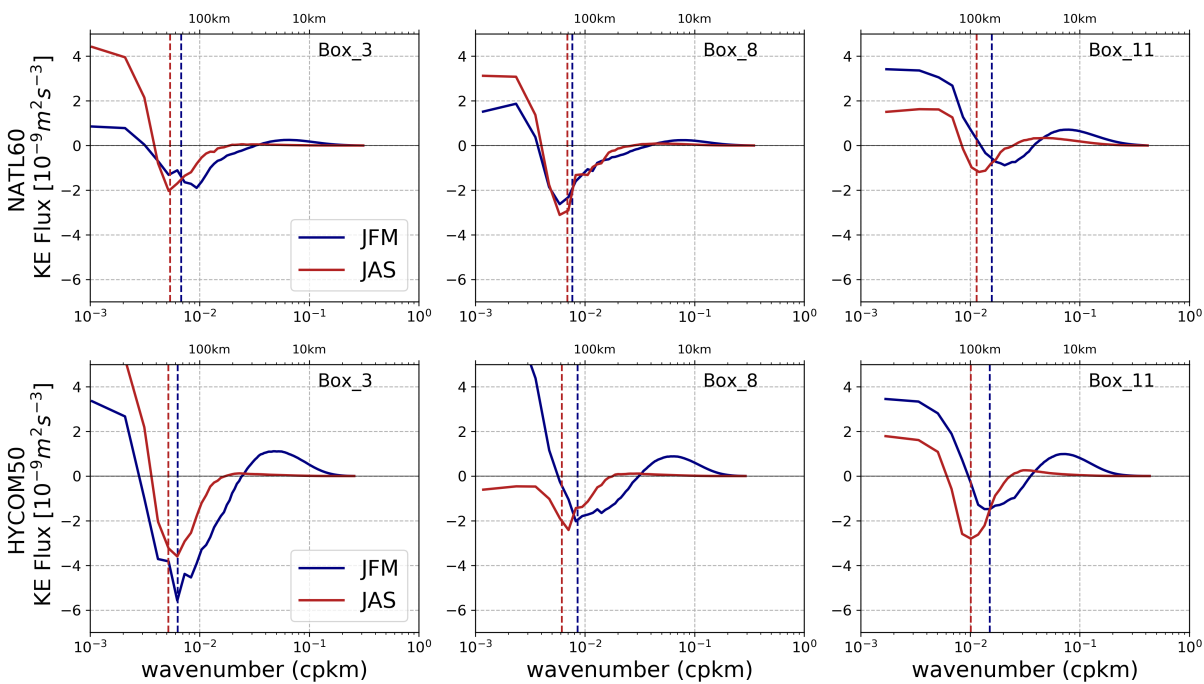

Figure 15. Winter (blue line) and summer (red line) average of surface kinetic energy spectral flux computed from daily output of horizontal total velocities. Dash lines represents the energy containing scale. NATL60 (upper panel) and HYCOM50 (lower panel) 

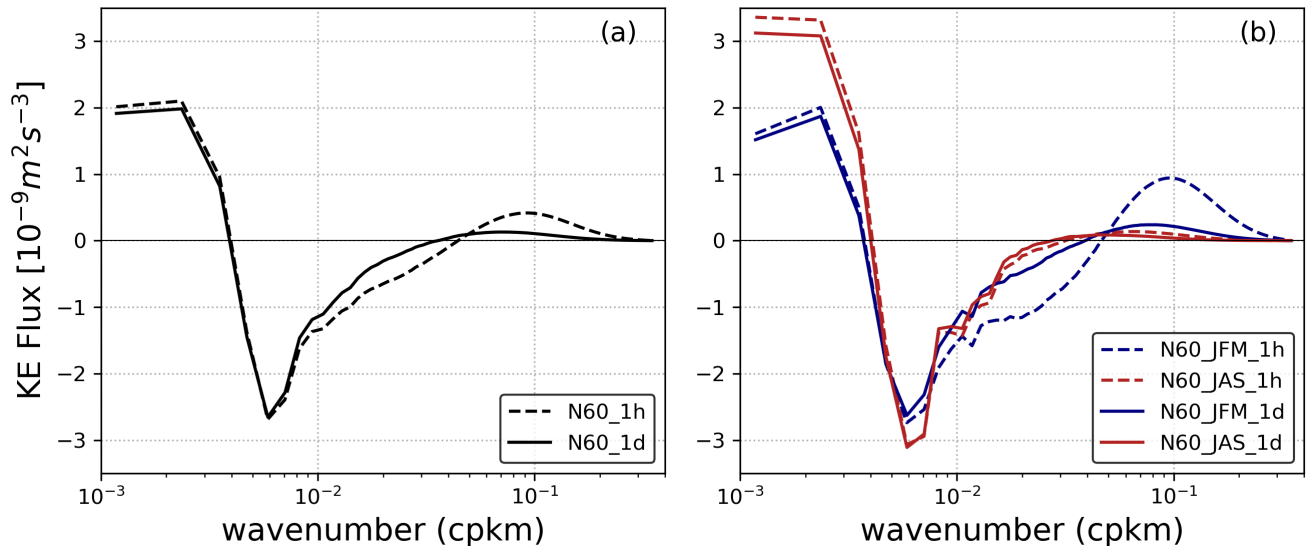

Figure 16. Comparison between surface kinetic energy spectral flux computed from daily averages (thick line) and hourly averages (dash line) of velocity outputs for Box 8. (a) one year mean (b) winter (blue line) and summer (red line) averages 

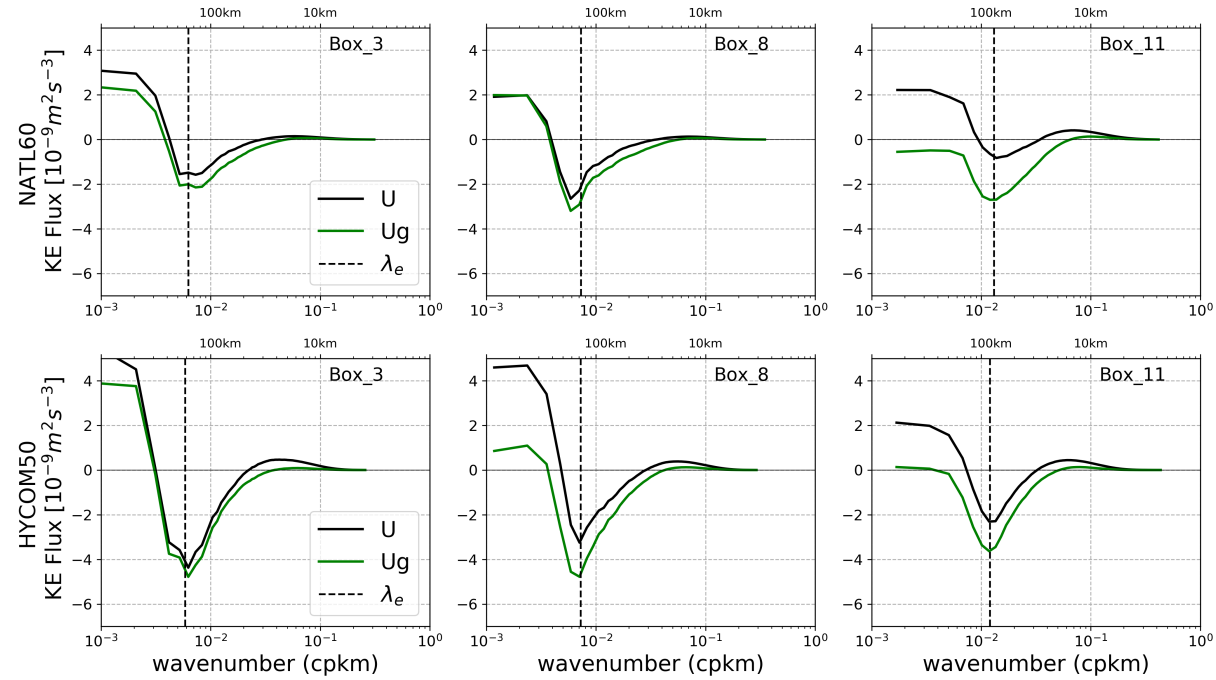

Figure 17. Surface kinetic energy spectral flux computed from total velocity (black line) versus geostrophic velocity (green line). NATL60 (upper panel) and HYCOM50 (lower panel) 


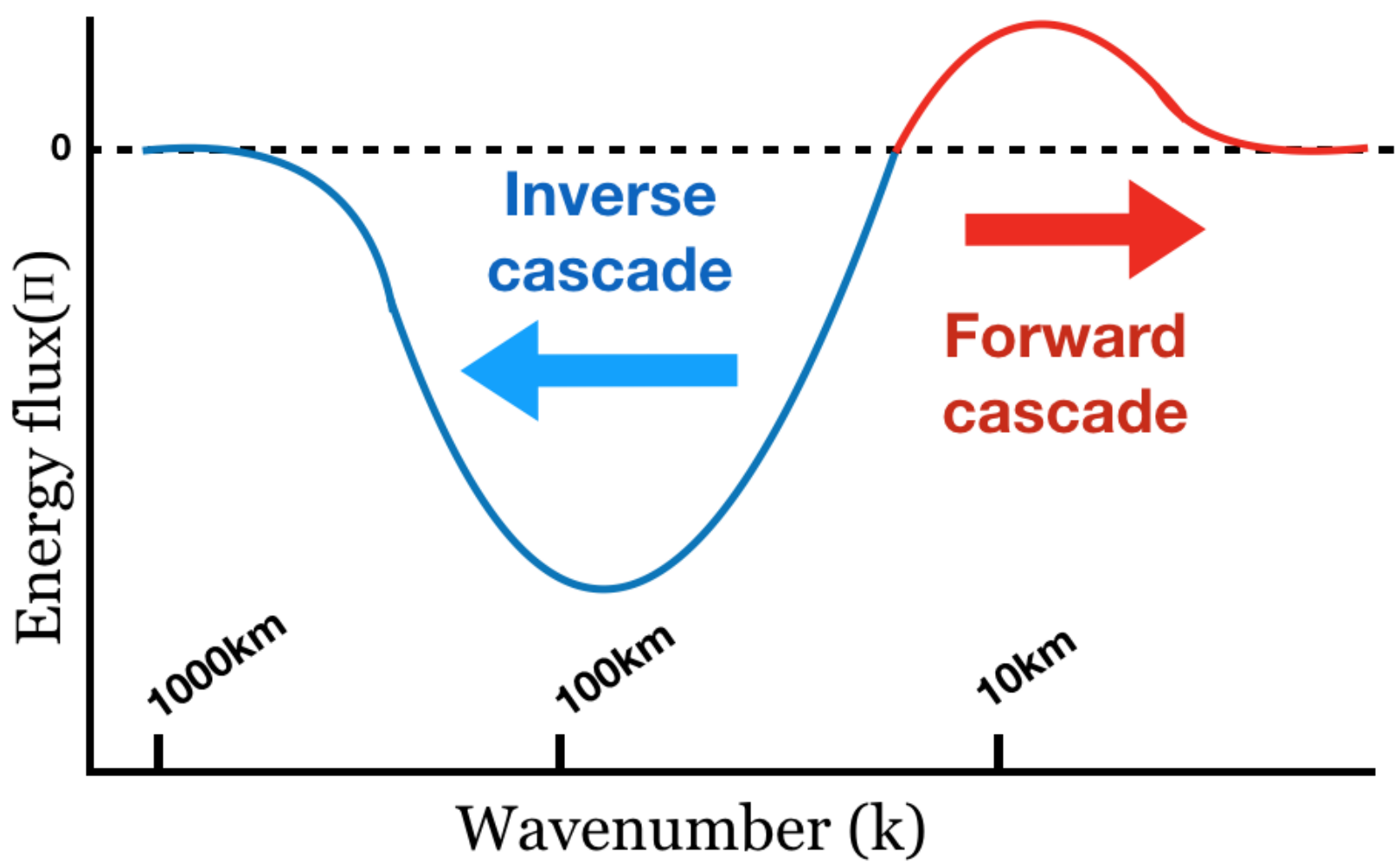

This article is protected by copyright. All rights reserved. 
Surface Speed
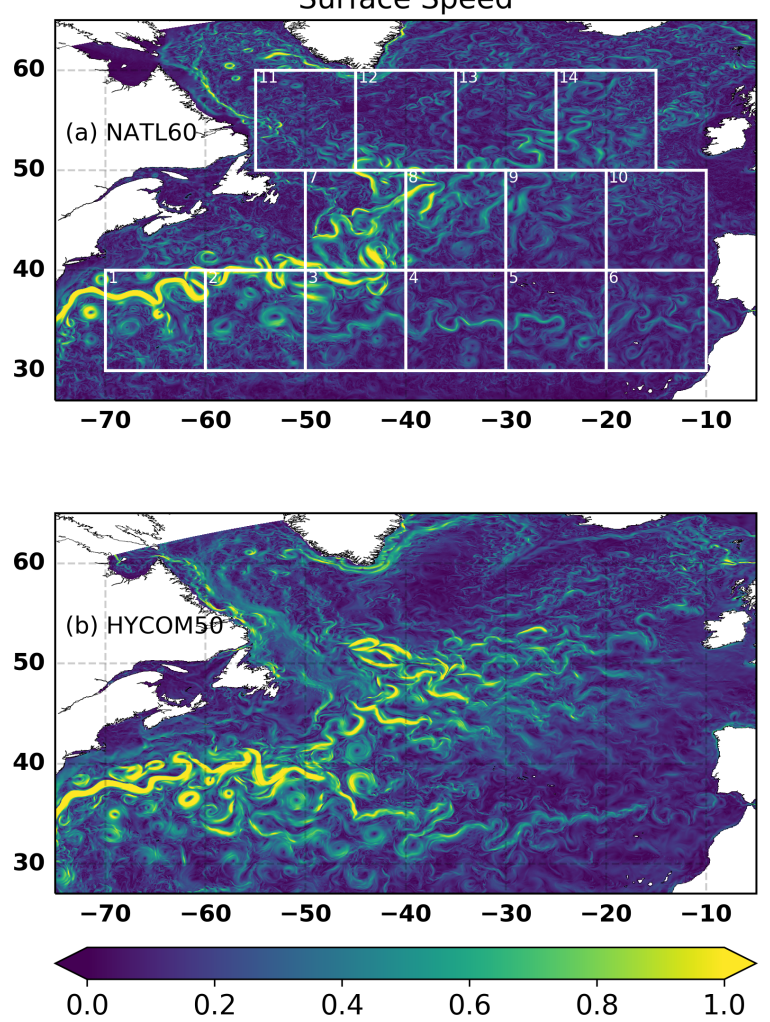
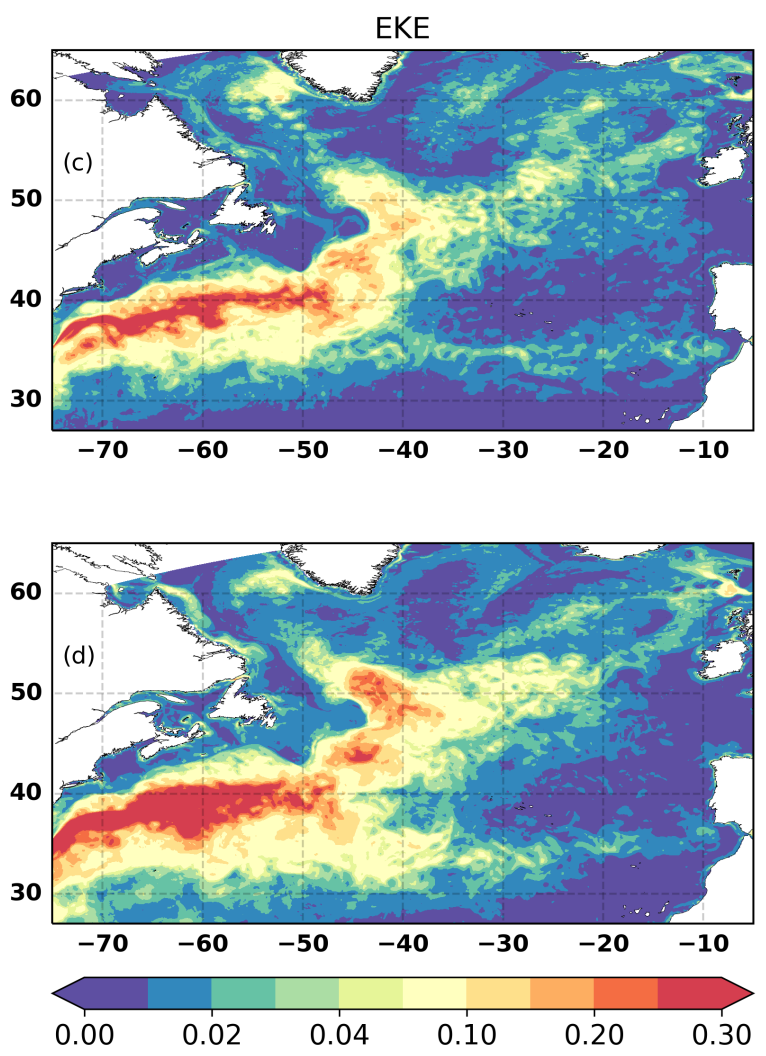

This article is protected by copyright. All rights reserved. 


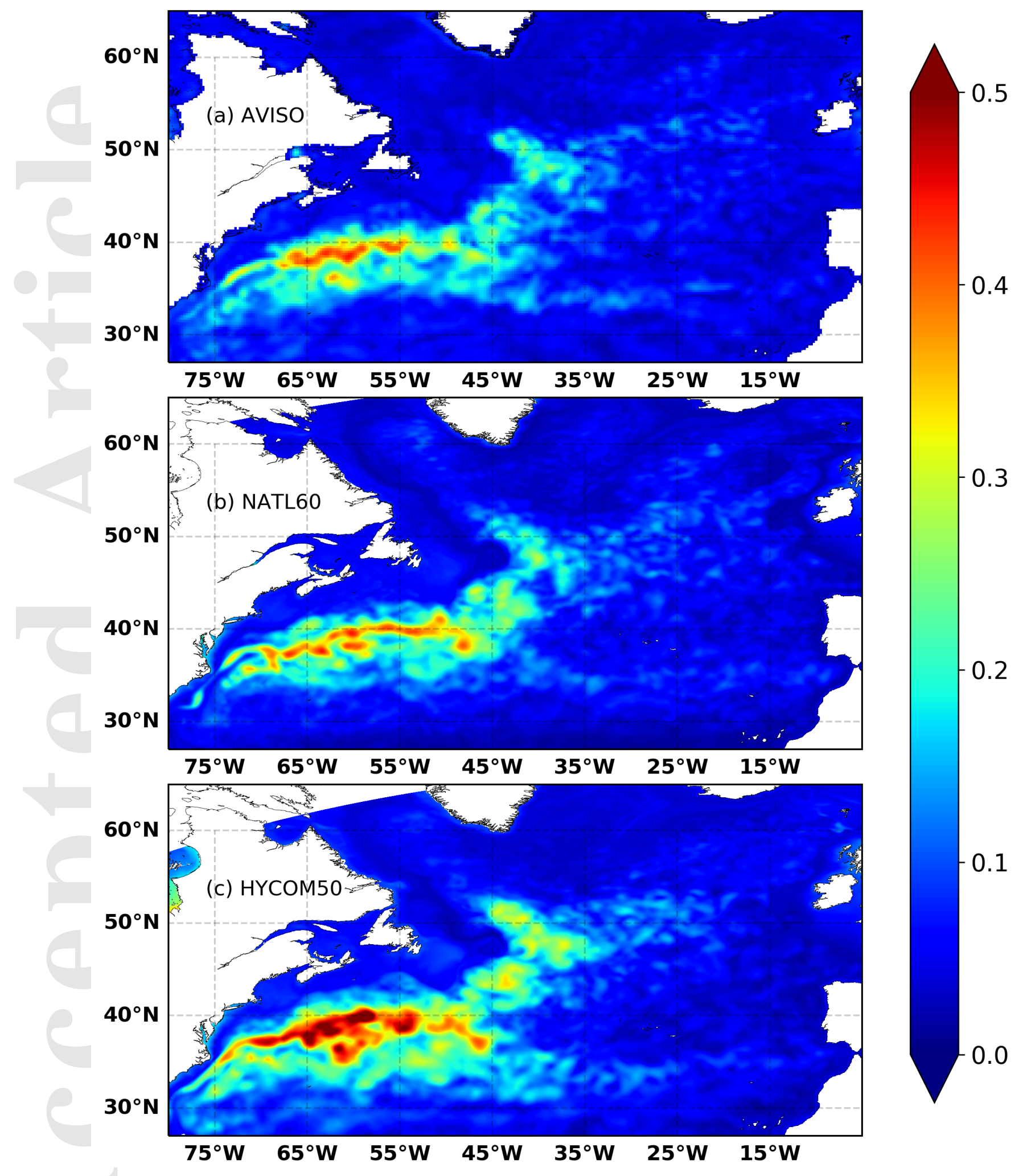

This article is protected by copyright. All rights reserved. 


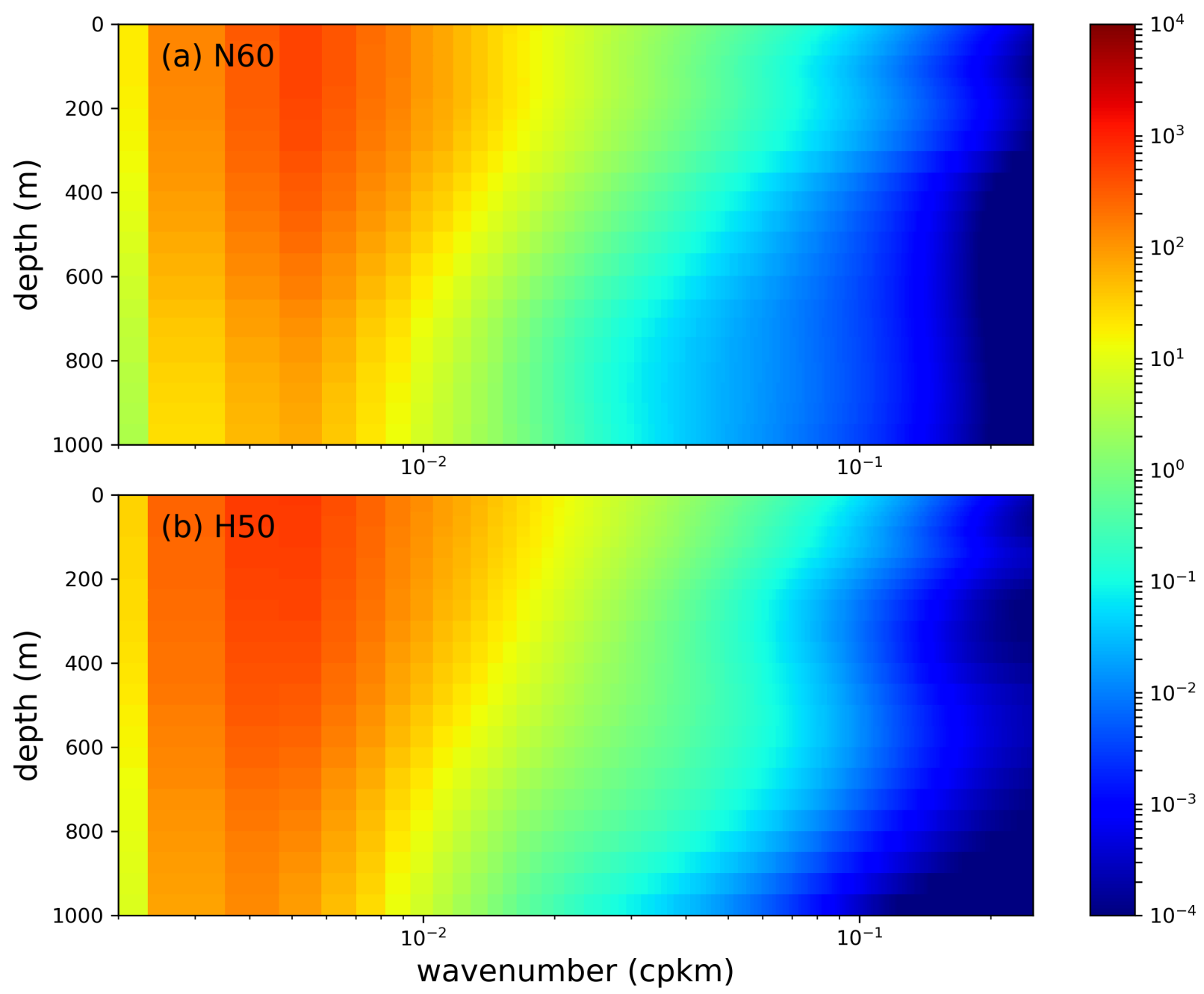

This article is protected by copyright. All rights reserved. 

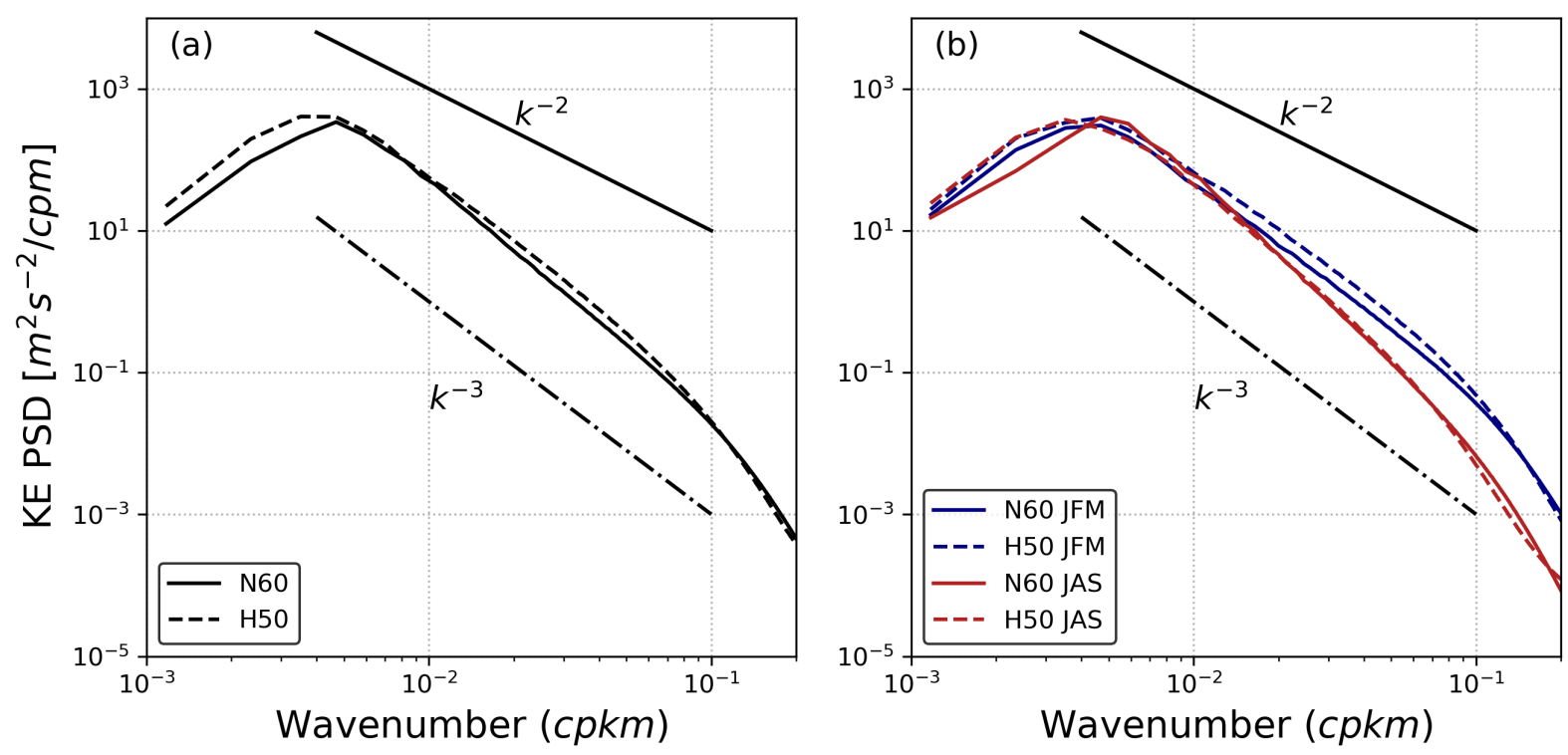

This article is protected by copyright. All rights reserved. 

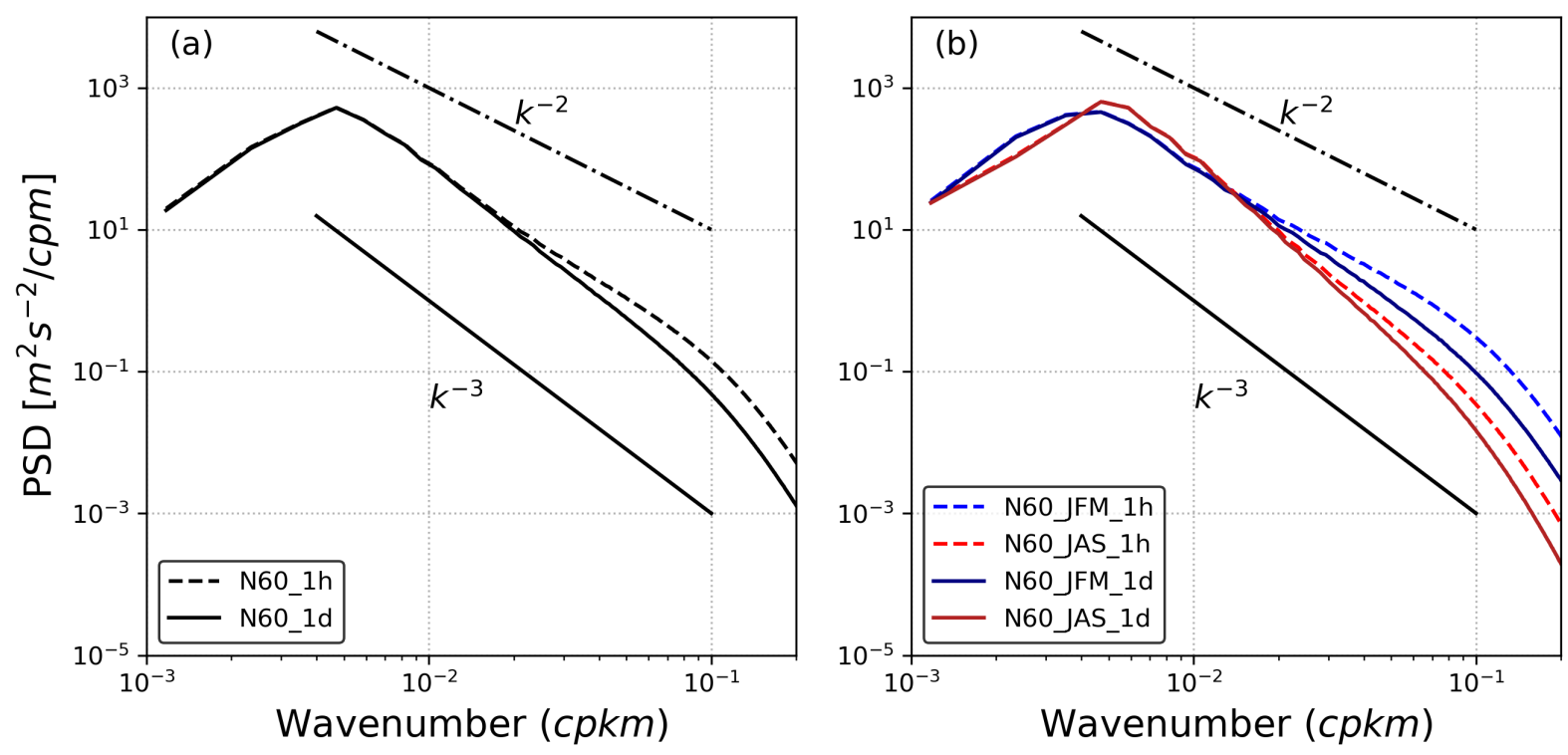

This article is protected by copyright. All rights reserved. 

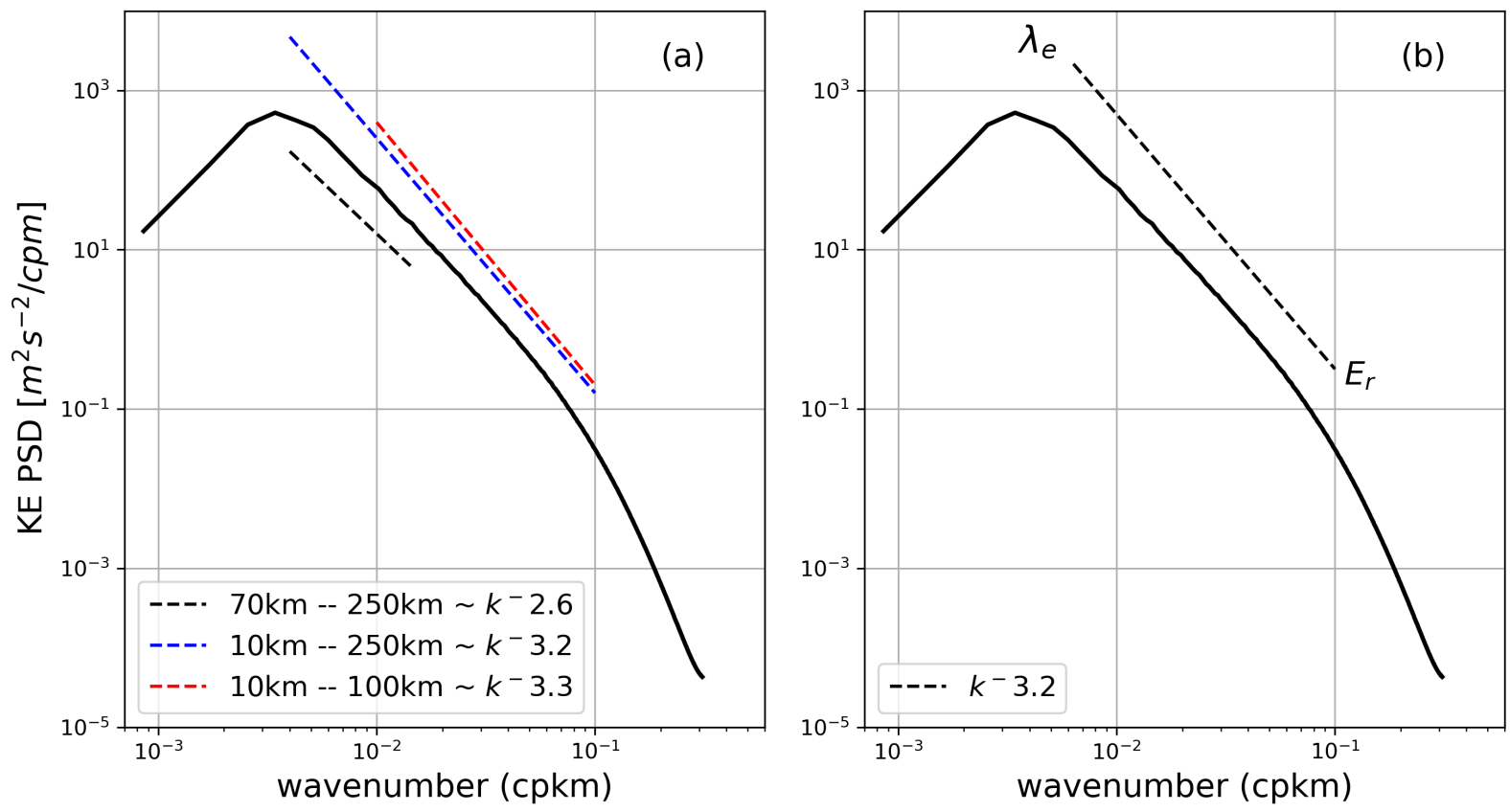

This article is protected by copyright. All rights reserved. 

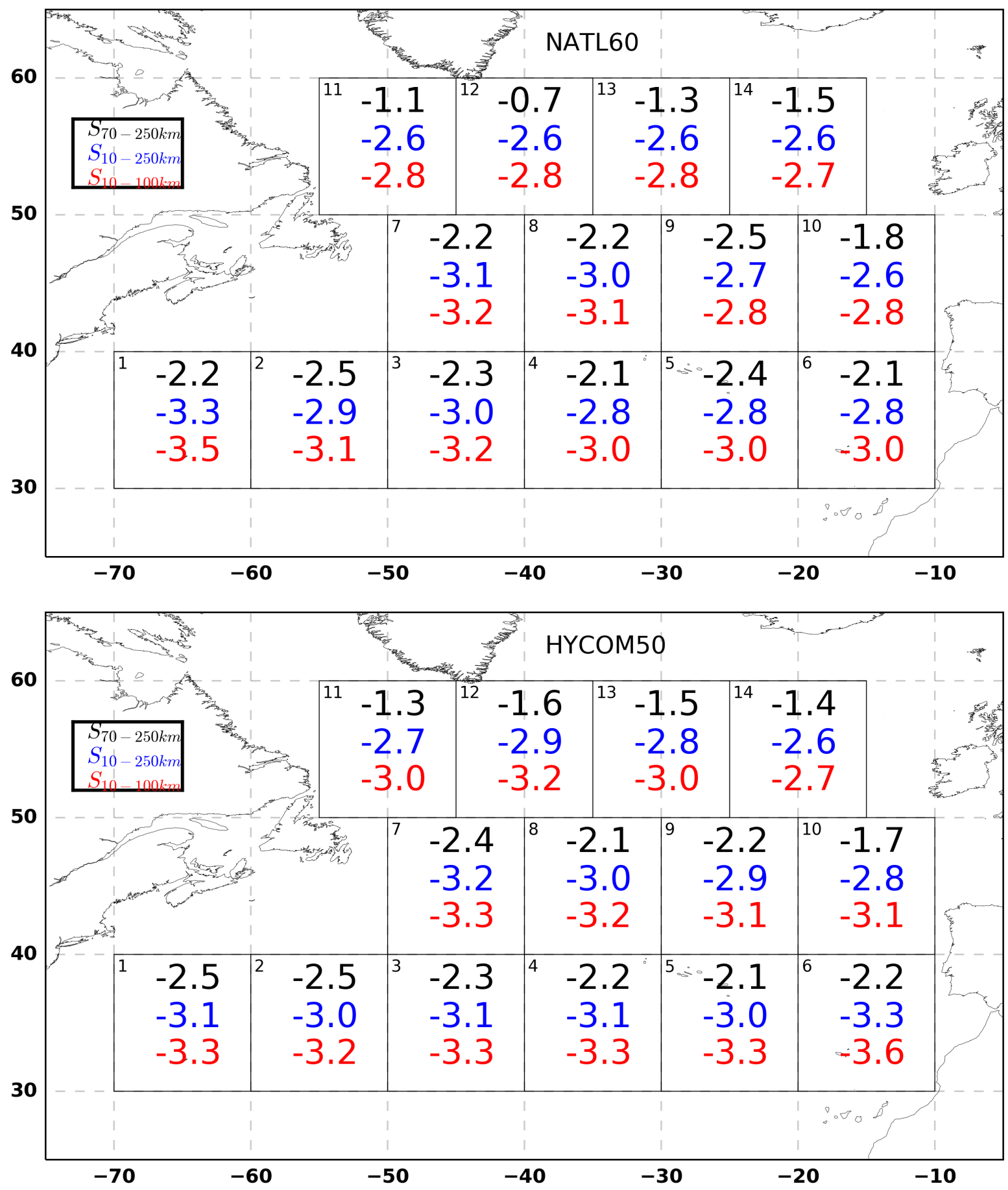

This article is protected by copyright. All rights reserved. 

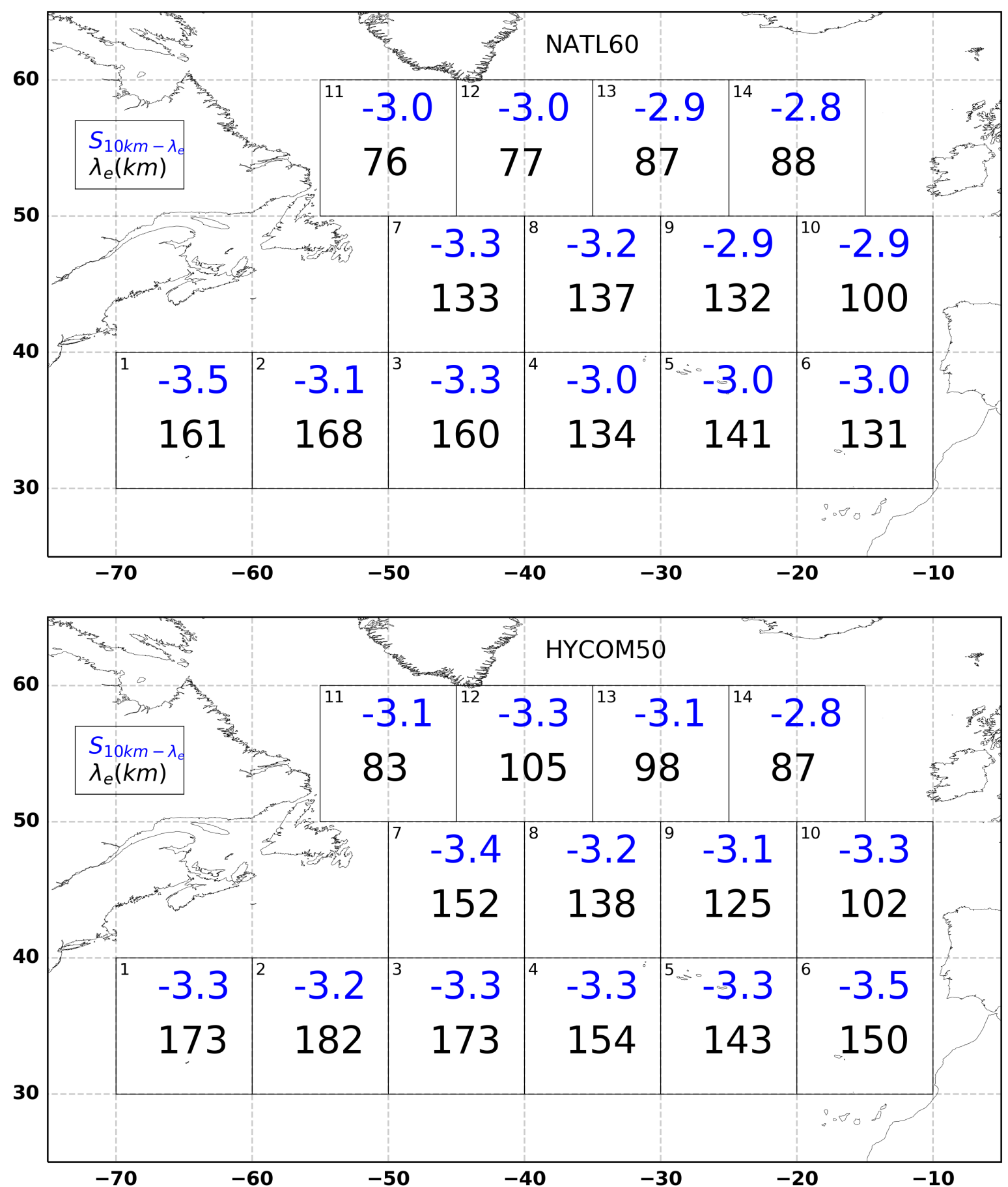

This article is protected by copyright. All rights reserved. 


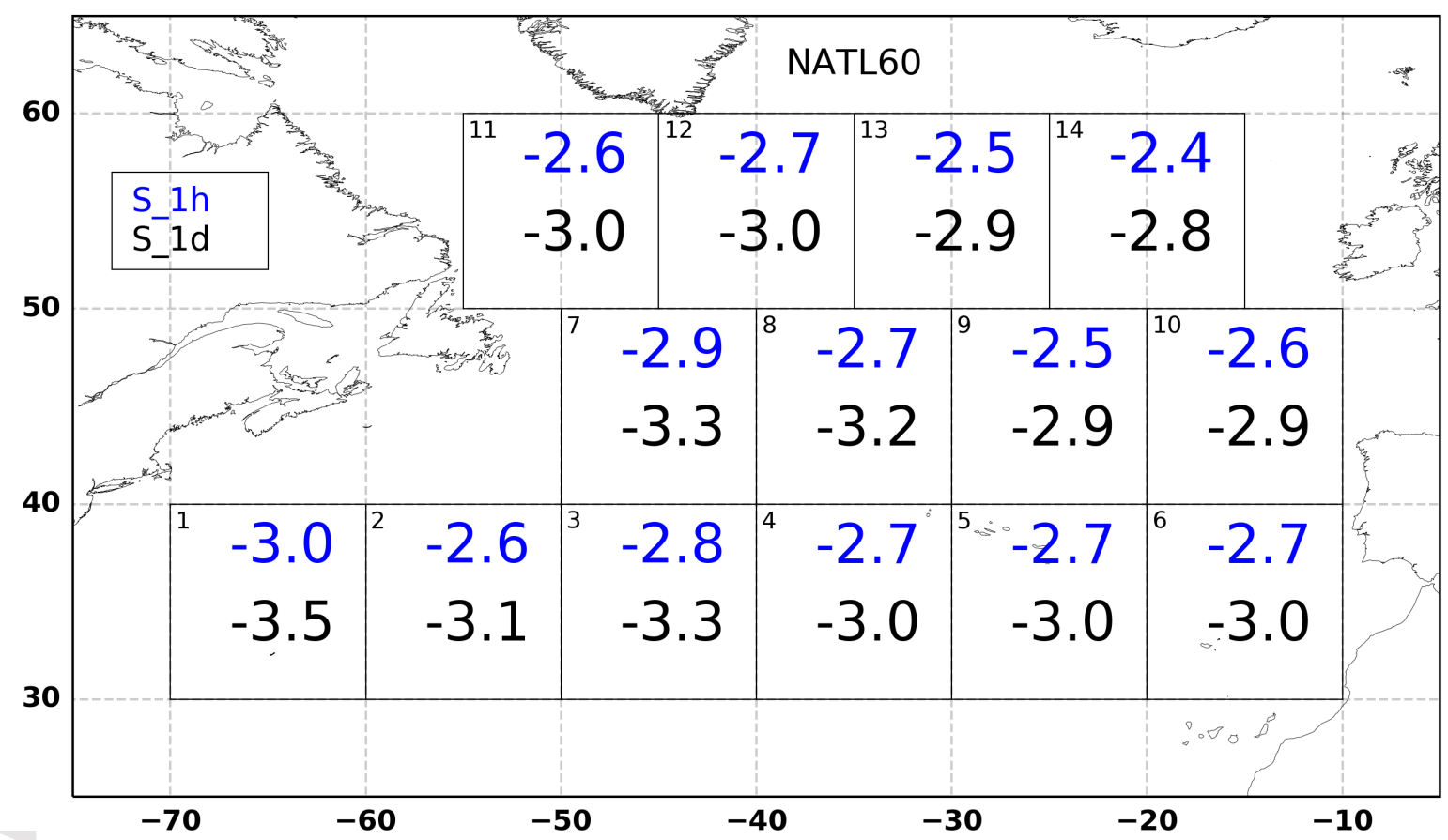

This article is protected by copyright. All rights reserved. 


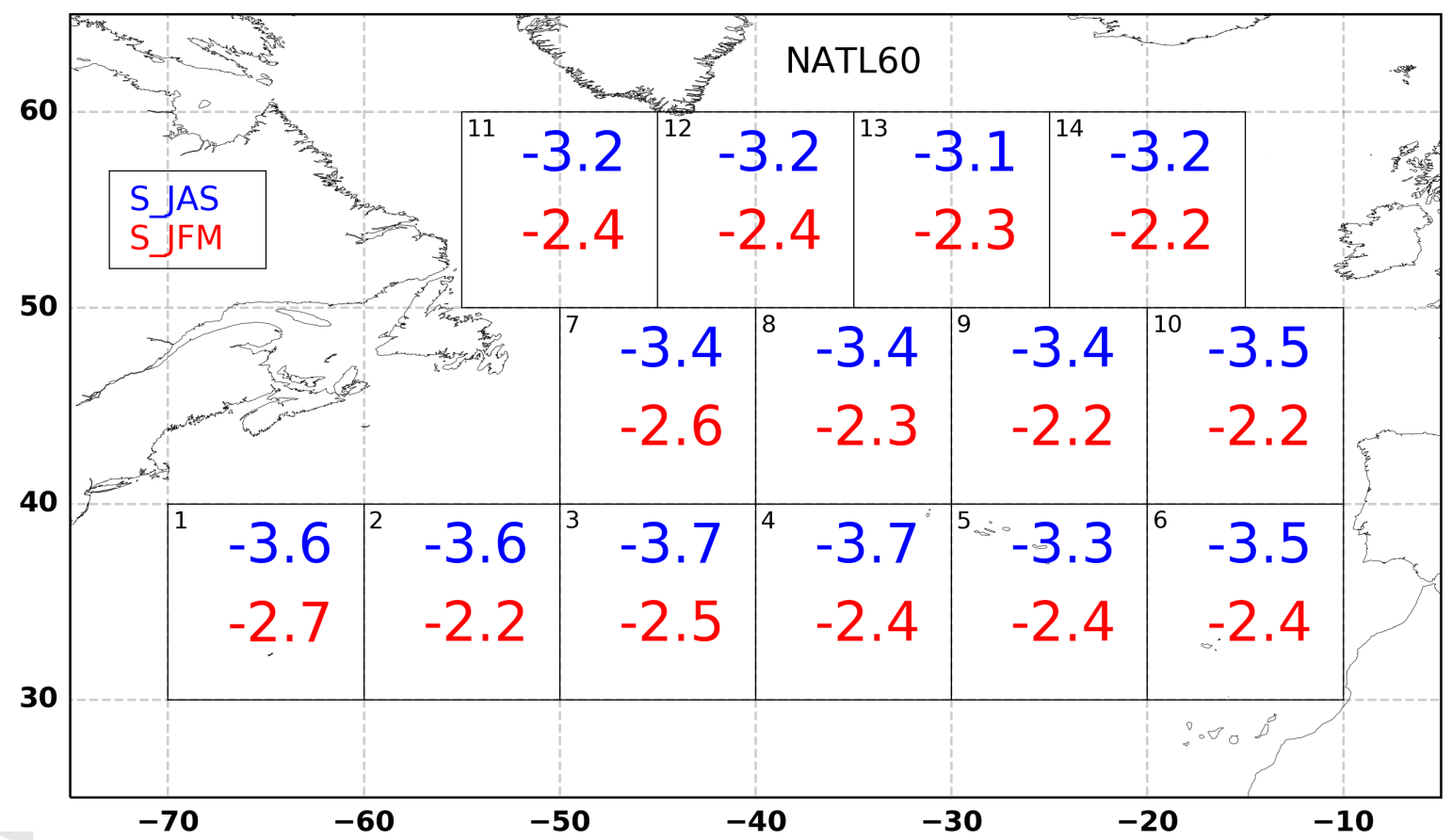

This article is protected by copyright. All rights reserved. 

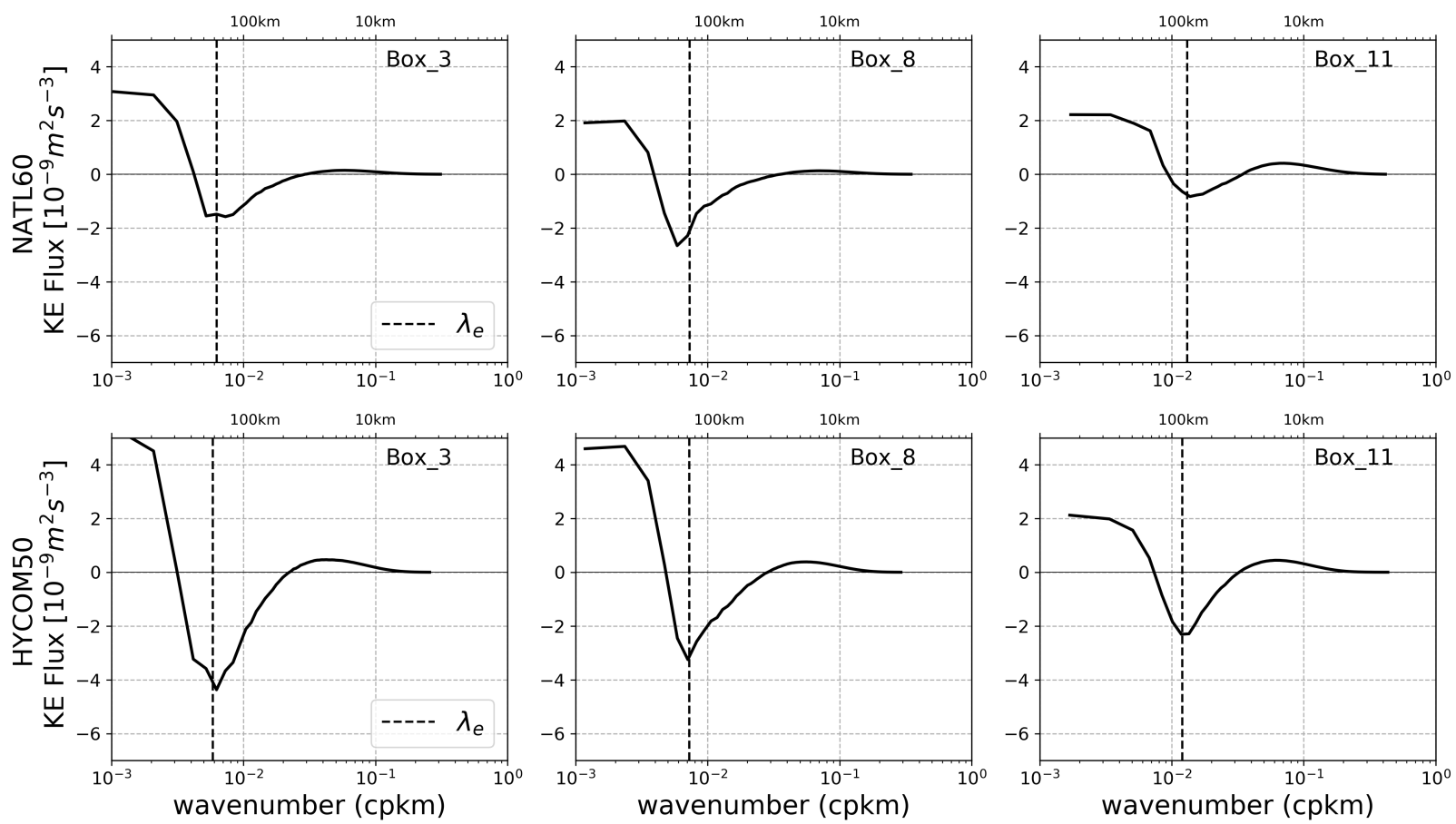

This article is protected by copyright. All rights reserved. 


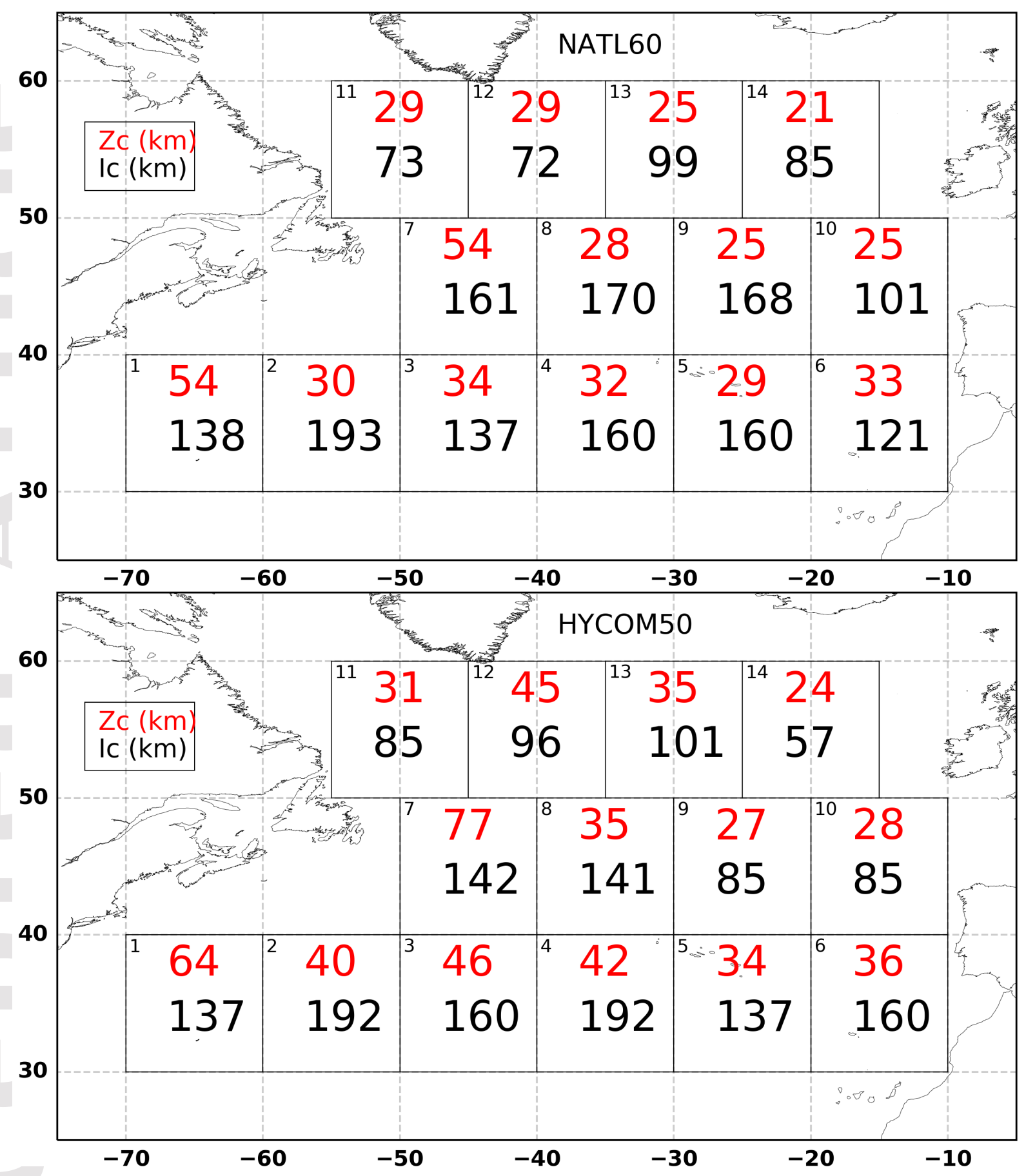

This article is protected by copyright. All rights reserved. 


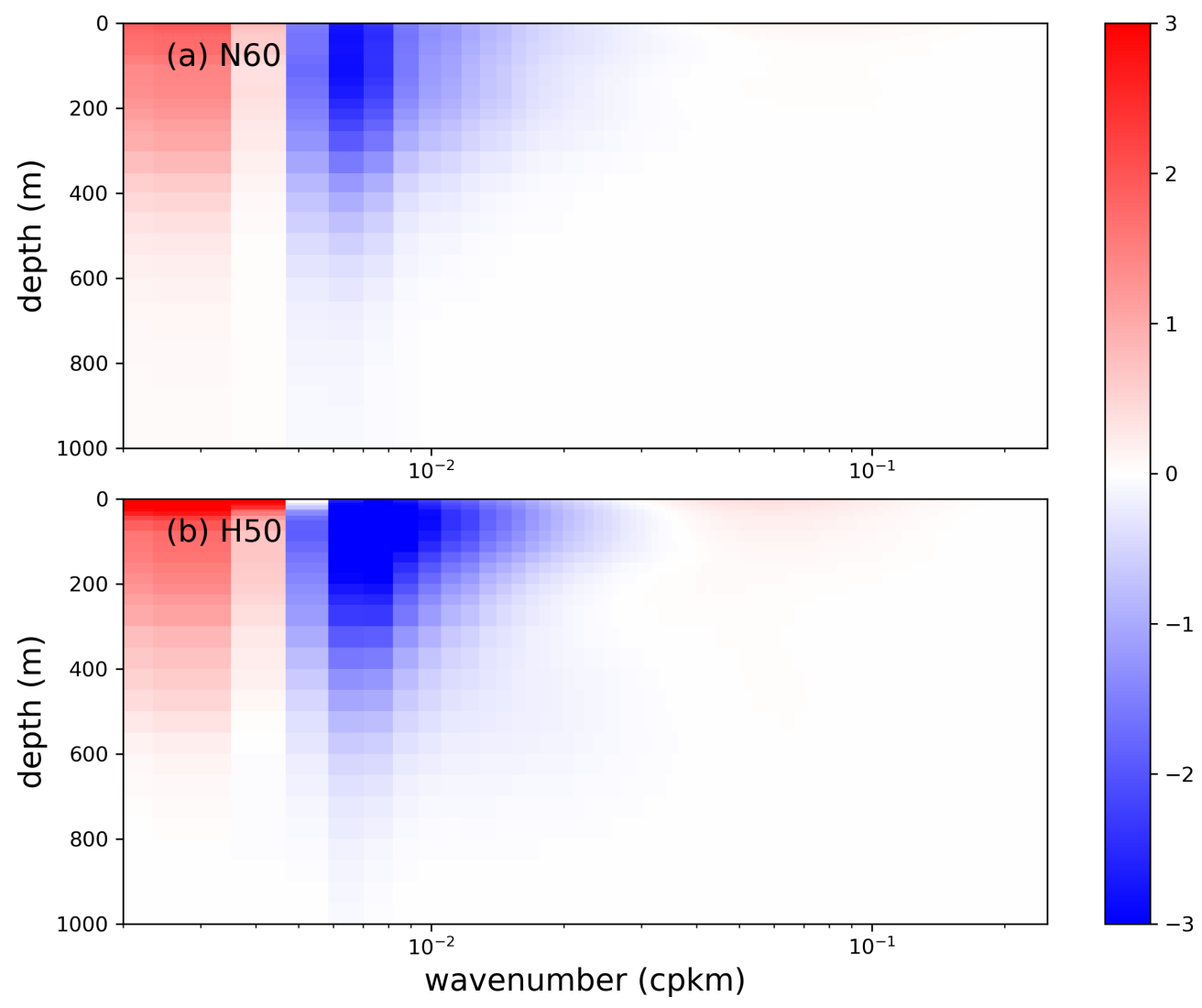

This article is protected by copyright. All rights reserved. 

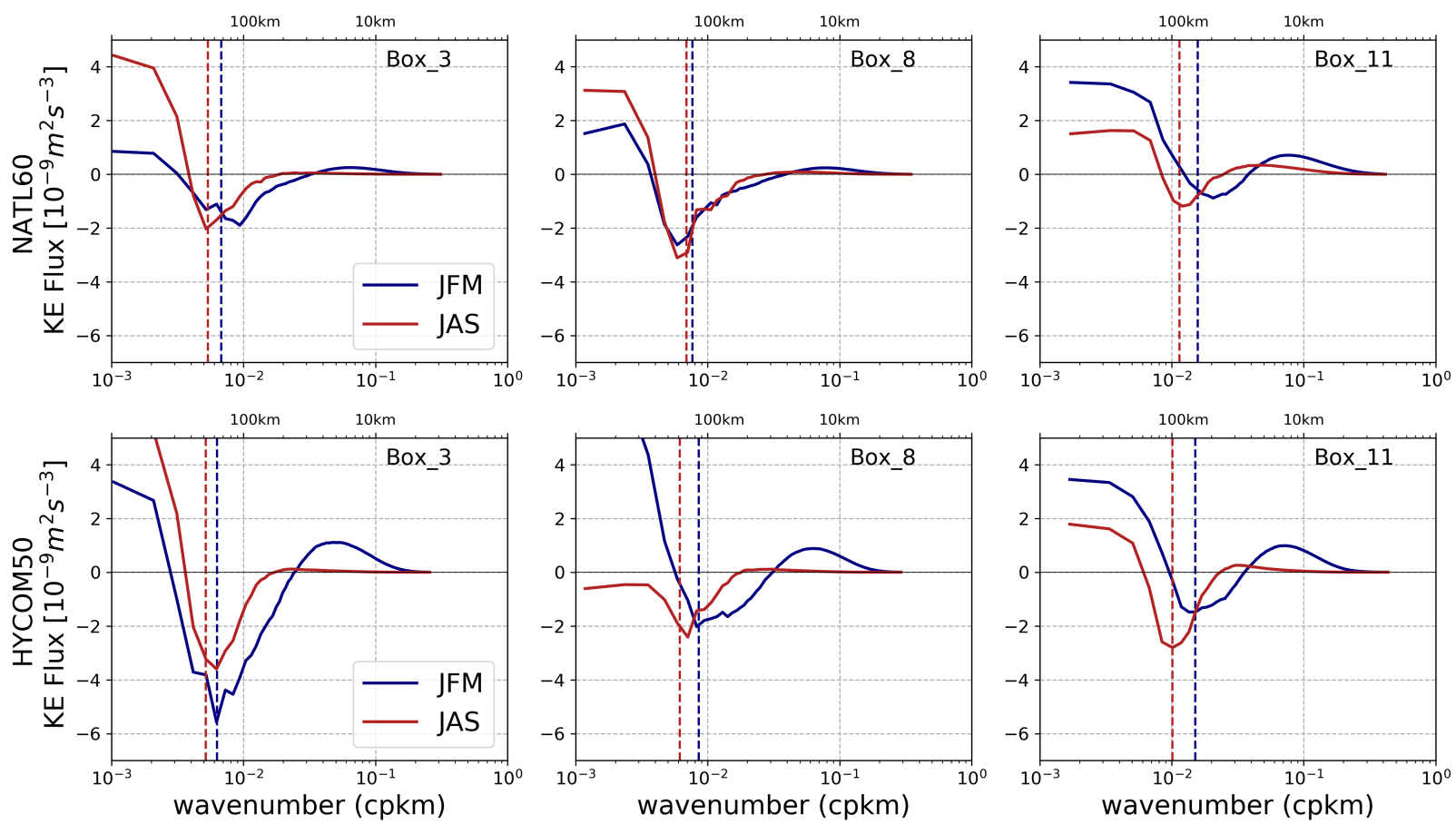

This article is protected by copyright. All rights reserved. 

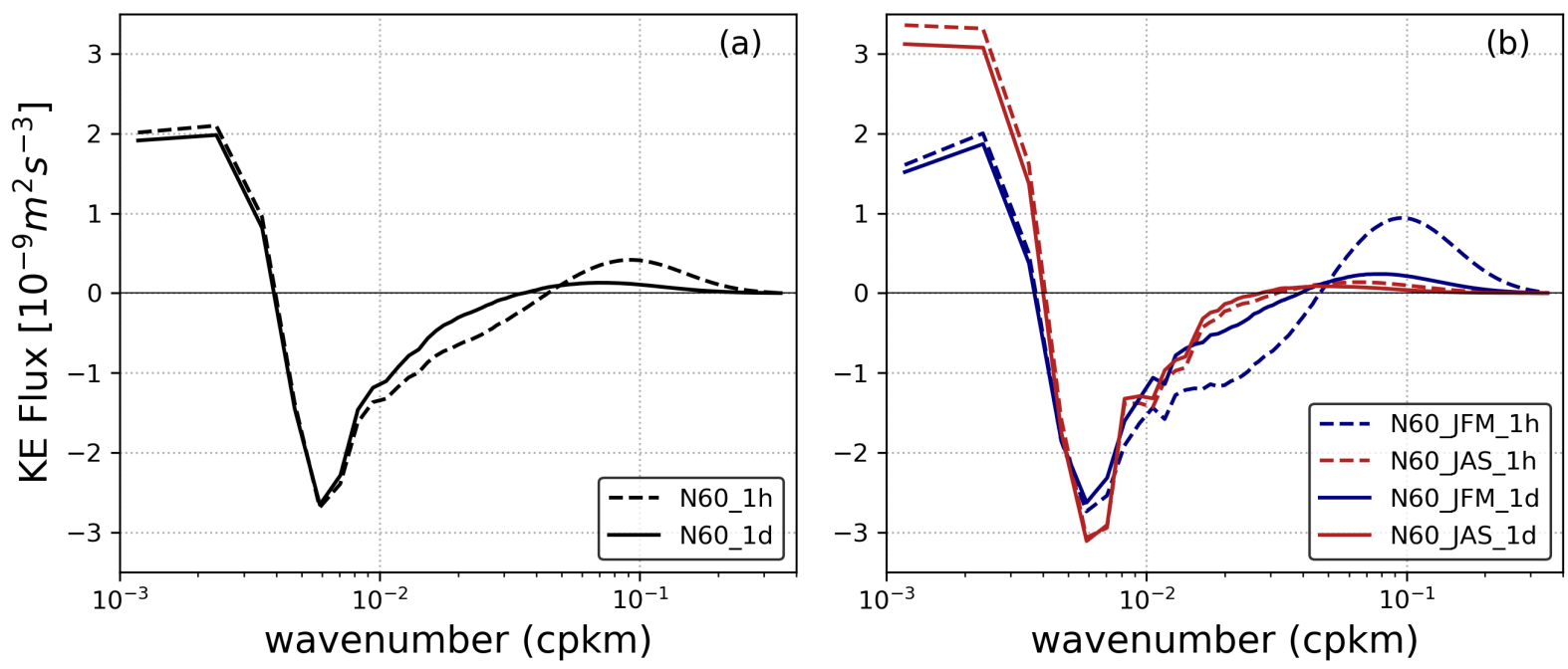

This article is protected by copyright. All rights reserved. 

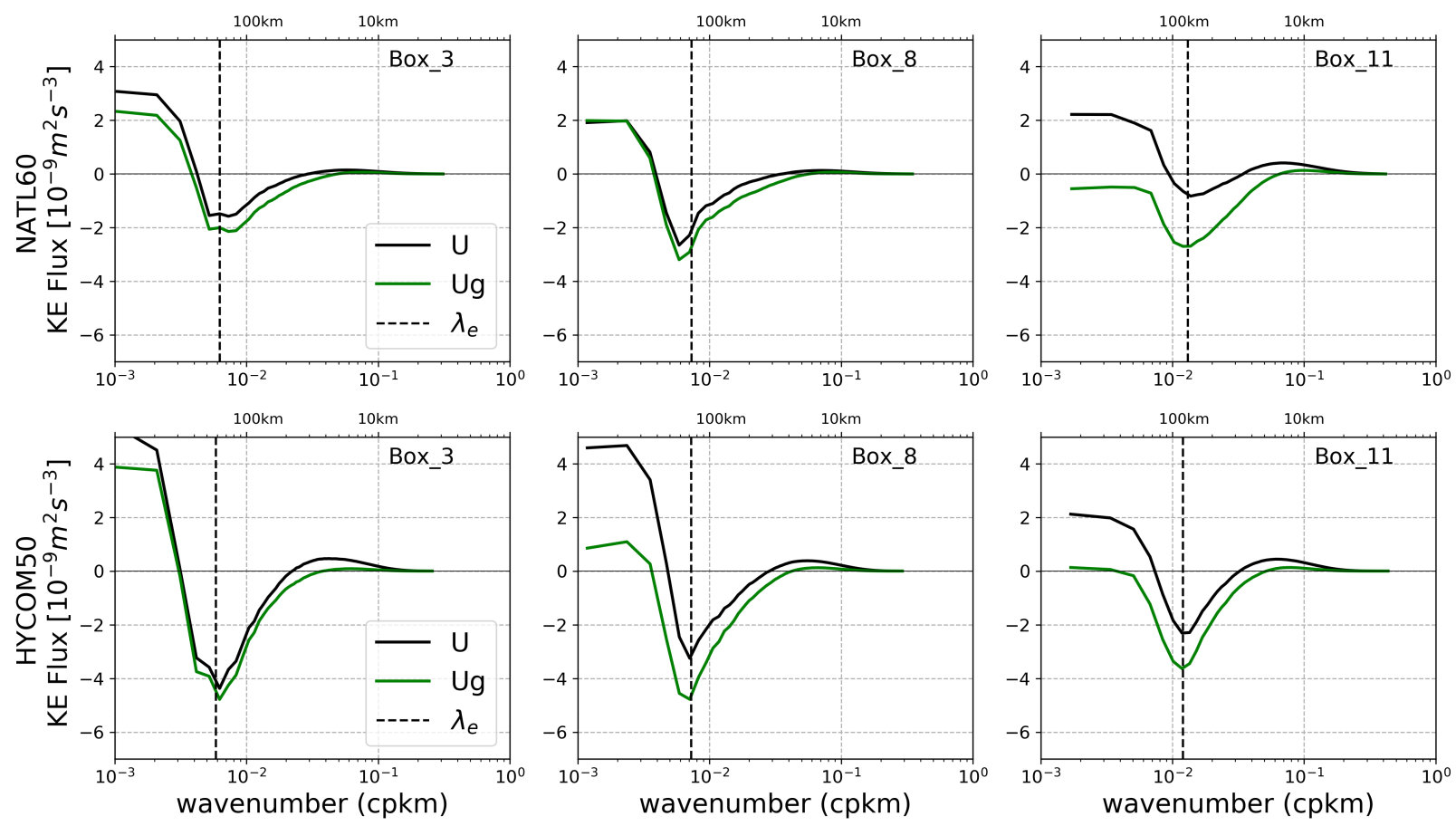

This article is protected by copyright. All rights reserved. 